Research Article

\title{
Optimal Selling Strategies under Regime-Switching Market Environment with Finite Expiry
}

\author{
Jie Xing $\mathbb{( i D}^{1}$ and Taoshun $\mathrm{He} \mathbb{1}^{2,3}$ \\ ${ }^{1}$ School of Big Data Applications and Economics (Guiyang Institute for Big Data and Finance), \\ Guizhou University of Finance and Economics, Guiyang 550025, China \\ ${ }^{2}$ Numerical Simulation Key Laboratory of Sichuan Province, College of Mathematics and Information Science, \\ Neijiang Normal University, Neijiang 641110, China \\ ${ }^{3}$ Institute for Studies in Mathematical Finance, College of Mathematics and Information Science, Neijiang Normal University, \\ Neijiang 641100, China
}

Correspondence should be addressed to Taoshun He; hstlcd@163.com

Received 9 June 2021; Revised 11 August 2021; Accepted 31 August 2021; Published 30 September 2021

Academic Editor: Xiaohua Ding

Copyright (C) 2021 Jie Xing and Taoshun He. This is an open access article distributed under the Creative Commons Attribution License, which permits unrestricted use, distribution, and reproduction in any medium, provided the original work is properly cited.

\begin{abstract}
This paper addresses an optimal stock liquidation problem over a finite-time horizon; to that end, we model it as an optimal stopping problem in a regime-switching market. The optimal stopping time is written as a solution to a system of Volterra type integral equations. Moreover, it reveals that when the risk-free interest rate is always lower than the return rate of the stock, it is never optimal to sell the stock early; otherwise, one should sell the stock in bear market if the stock price reaches a critical value and hold the stock in bull market until the maturity date. Finally, we present a trinomial tree method for numerical implementation. The numerical results are consistent with the theoretical findings.
\end{abstract}

\section{Introduction}

"What is the best time to sell an asset to maximize revenue?" is a fundamental problem in finance. Given a fixed transaction cost, the objective is to choose a stopping time so as to maximize an expected return. This kind of optimal stopping problem was studied by McKean firstly under Black-Scholes model [1]. It is well known that the stochastic variability in the market parameters is not reflected in the Black-Scholes model. At present, the market trend is considered in literature when dealing with the optimal stock liquidation problem. In [2], Wang et al. proposed a Markov regimeswitching DCC-GARCH model and estimated the model parameters by a two-stage maximum likelihood method. Hou et al. presented a spatial-temporal neural network frame to forecast the stock price movement [3].

In [4], Zhang provided a suboptimal selling rule in a regime-switching market by a two-point-boundary approach. For regime-switching exponential Gaussian diffusion model, Eloe et al. used a similar approach to derive an optimal selling rule by two threshold levels [5]. Guo and Zhang used a smooth-fit technique to analyze the optimal selling boundary under certain parameter conditions for infinite horizontal problem [6]. The finite horizontal optimal selling rule under regime-switching model was firstly studied by Pemy and Zhang using viscosity solution approach [7]. Later, Pemy studied a similar problem in the case where the underlying dynamic is a Markov switching Lévy process [8]. Bian et al. investigated the optimal liquidation problem with regime-switching until the exit time, which is essentially an optimal control problem rather than an optimal stopping problem [9]. Recently, Vaicenavicius put forward an optimal liquidation problem involved with drift uncertainty and regime-switching volatility and solved it by constructing approximating sequences of optimal stopping problem with constant volatility [10].

It is known that Pemy and Zhang only discussed the value function to the related $\mathrm{HJB}$ equation, and up to the 
author's knowledge, the optimal selling strategies under regime-switching model are still unknown so far [7]. In filling this gap, this paper is concerned with the optimal selling strategies. That is, we mainly focus on the early exercise boundary with respect to the optimal stopping problem rather than the value function. Indeed, the role of the present paper should be viewed as a supplement to [7]. On the other hand, the impacts of varying parameters on the selling decision were not discussed in [7]. We discuss it rigorously by variational inequality approach. It is important to distinguish this paper from the existing literature on optimal stopping problems with regime-switching volatility. Buffington and Elliott discussed American options in regime-switching market and obtained an approximate valuation of American option [11]. Jacka and Ocejo studied the regularity of the value function for a finite horizontal optimal stopping problem in the presence of regime-switching uncertainty [12]. The abovementioned authors mainly concentrated on the value function and did not investigate the free boundary arising from American option pricing problem. Boyarchenkp and Levendorski calculated the early exercise boundaries for American options by using a generalization of Carr's randomization procedure for regimeswitching models [13]. But they did not study the properties of the free boundary and the early exercise premium was unknown. Liu and Privault dealt with optimal prediction problem in a regime-switching model and derived Volterra type integral equations [14]. Since the optimal stopping problem involves with a system of interacting integral equations, making it more difficult to solve directly, they solved the optimal stopping problem by using a recursive algorithm later and their method did not rely on the Volterra equations [10]. Recently, Luo and Xing handled an optimal stopping problem in pricing variable annuities under regime-switching volatility, but the drift term in the model was assumed to be constant [15].

In the above optimal stopping problems, there exists a unique free boundary for each state in common, whereas the existence of early exercise boundary in our problem depends on the parameters in the model. Furthermore, since there has been less focus on the development of a realistic modelling framework for analyzing the impact of various sources of risk in influencing the early exercise boundary, it is crucial to make rigorous sensitivity analysis when we make decisions. The main contribution of this paper is that we discuss the optimal selling decisions rigorously, which is not investigated in [7]. Specifically, the contributions are threefold: properties of the early exercise boundary, regimeswitching Volterra type integral equations, and sensitivity analysis of optimal selling strategy. Firstly, by using the properties of value function, we derive some basic properties of free boundary. Then, by dynamic programing principle, we convert the regime-switching optimal stopping problem into an optimal stopping problem with constant-coefficient geometric Brownian motion, and the proof is novel compared with the method used in [14]. Finally, we use a variational inequality approach inspired by [16] to derive sensitivity analysis with respect to the model parameters and the numerical results are consistent with the theorems.
The remainder of this paper is organized as follows. In Section 2, we formulate the optimal selling problem in a regime-switching market with finite expiry. In Section 3, we give some preliminary results which will be frequently used later. Section 4 presents the main results, namely, the properties of free boundary, integral equation for the free boundary, and sensitivity analysis of the free boundary. In Section 5, we use the trinomial tree method mentioned in [17] to calculate the optimal stopping problem and perform some numerical experiments. Finally, Section 6 concludes.

\section{Problem Set-Up}

Consider a probability space $(\Omega, \mathscr{F}, \mathbb{P})$. Consider also a standard Brownian motion $B=\left\{B_{t}, t \geq 0\right\}$. We assume that the continuous time Markov chain $\alpha=\left\{\alpha_{t}, t \geq 0\right\}$ takes values in $\mathcal{S}=\{1,2\}$, i.e. there are only two states: "bull" and "bear," and the generator of $\alpha_{t}$ is of the form

$$
A=\left(\begin{array}{cc}
-\lambda_{1} & \lambda_{1} \\
\lambda_{2} & -\lambda_{2}
\end{array}\right),
$$

where $\lambda_{1}$ and $\lambda_{2}$ are the positive constants. We denote by $\mathbb{F}=\left\{\mathscr{F}_{t}, t \geq 0\right\}$ the $\mathbb{P}$-augmentation of the filtration $\left\{\mathscr{F}_{t}^{B, \alpha}, t \geq 0\right\}$ generated by the stochastic process $B$ and $\alpha$. Here, $\left\{\mathscr{F}_{t}^{B, \alpha}:=\sigma\left\{B_{v}, \alpha_{v}: 0 \leq v \leq t\right\}\right.$ for $t \in[0,+\infty)$. Moreover, the stock price satisfies the following Markovian switching stochastic differential equation:

$$
\begin{aligned}
\mathrm{d} X_{s} & =X_{s}\left(\mu\left(\alpha_{s}\right) \mathrm{d} s+\sigma\left(\alpha_{s}\right) \mathrm{d} B_{s}\right), \quad s>t, \\
X_{t} & =x,
\end{aligned}
$$

where $x$ is the initial stock price, $\mu\left(\alpha_{s}\right) \in\left\{\mu_{1}, \mu_{2}\right\}$ is the rate of return, and $\sigma\left(\alpha_{s}\right) \in\left\{\sigma_{1}, \sigma_{2}\right\}$ is the volatility.

Let $\Lambda_{t, T}$ denote the set of all $\mathscr{F}_{t}$-stopping times taking value in $[t, T]$ a.s. Given a transaction cost $a>0$, the agent aims to maximize $\mathbb{E}\left[e^{-r(\tau-t)}\left(X_{\tau}-a\right)\right]$ by choosing a stopping time $\tau$, where $r$ is the risk-free interest rate. Therefore, we consider the optimal stopping problem as follows:

$$
\begin{array}{r}
V(t, x, i)=\sup _{\tau \in \Lambda_{t, T}} \mathbb{E}\left[e^{-r(\tau-t)}\left(X_{\tau}^{t, x}-a\right) \mid X_{t}=x, \alpha_{t}=i\right], \\
i=1,2,
\end{array}
$$

where $X^{t, x}=\left\{X_{s}^{t, x}, s \geq t\right\}$ is the strong solution to the $\operatorname{SDE}$ (2) starting from $x$ at time $t$.

Denote $g(x)=x-a$. By Markov property of $X$, we can rewrite the optimal stopping problem as follows:

$$
V(t, x, i)=\sup _{\tau \in \Lambda_{0, T-t}} \mathbb{E}^{i, x}\left[e^{-r \tau} g\left(X_{\tau}^{x}\right)\right], \quad i \in \mathcal{S},
$$

where $X^{x}=X^{0, x}$ is the strong solution to the SDE (2) starting from $x$ at time 0 and $\mathbb{E}\left[\cdot \mid X_{0}=x, \alpha_{0}=i\right]:=\mathbb{E}^{i, x}[\cdot]$. For simplicity, we will always omit the superscripts in $\mathbb{E}^{i, x}$.

Besides, we assume that the following assumption holds throughout this paper.

Assumption 1. The rate of return satisfies $\mu(1)=\mu_{1}<$ $\mu_{2}=\mu(2)$. 


\section{Some Preliminary Results}

The following notations will be used throughout this paper. Define the stopping region and continuation region as follows:

$$
\begin{aligned}
\mathscr{D} & :=\{(t, x, i) \in[0, T] \times(0,+\infty) \times \mathcal{S}: V(t, x, i)=g(x)\}, \\
\mathscr{C} & :=\{(t, x, i) \in[0, T] \times(0,+\infty) \times \mathcal{S}: V(t, x, i)>g(x)\}, \\
\mathscr{D}_{i} & :=\{(t, x) \in[0, T] \times(0,+\infty): V(t, x, i)=g(x)\}, \quad i \in \mathcal{S}, \\
\mathscr{C}_{i} & :=\{(t, x) \in[0, T] \times(0,+\infty): V(t, x, i)>g(x)\}, \quad i \in \mathcal{S} .
\end{aligned}
$$

We also introduce the infinitesimal generator of $\left(X_{t}, \alpha_{t}\right)$ as follows:

$$
(\mathscr{A} f)(t, x, i):=(\mathscr{L} f)(t, x, i)+\lambda_{i}(f(t, x, j)-f(t, x, i)), \quad j \neq i,
$$

where

$$
(\mathscr{L} f)(t, x, i):=\frac{1}{2} x^{2} \sigma_{i}^{2} f_{x x}(t, x, i)+x \mu_{i} f_{x}(t, x, i),
$$

where $f_{x}$ denotes $\partial f(t, x, i) / \partial x$, and $f_{x x}$ is defined similarly.

Lemma 1. The value function $V(t, x, i)$ is decreasing in $t$, increasing in $x$, and convex in $x$.

Proof. The proof is easy; we refer to Appendix A.

Next, we show that $V(\cdot, x, i)$ is $1 / 2-$ Hölder continuous and $V(t, \cdot, i)$ is Lipschitz continuous.

Lemma 2. For each $(x, i) \in \mathbb{R}^{+} \times \mathcal{S}$, the function $V(\cdot, x, i)$ is $1 / 2-H o ̈ l d e r$ continuous uniformly over a neighborhood of $x$; for each $(t, i) \in[0, T] \times \mathcal{S}$, the value function $V(t, \cdot, i)$ is Lipschitz continuous in $\mathbb{R}^{+}$. Moreover, it has at most linear growth rate, i.e., there exists a constant $C$ such that $|V(t, x, i)| \leq C(1+|x|)$.

Proof. For the proof, we refer to Appendix B.

It is clear that

$$
\mathbb{E}\left[\sup _{0 \leq s \leq T} e^{-r s}\left|X_{s}-a\right|\right] \leq \mathbb{E}\left[\sup _{0 \leq s \leq T} e^{-r s}\left|X_{s}\right|\right]+a<+\infty,
$$

where the last inequality follows from the moment estimates (see [18] or [12]). By Corollary 2.9 in [19], the above inequality together with the fact that $V$ and $g$ are continuous (Lemma 2) implies that

$$
\tau_{\mathscr{D}}(t, x, i):=\inf \left\{0 \leq s \leq T-t:\left(t+s, X_{s}^{x}, \alpha_{s}\right) \in \mathscr{D}\right\}
$$

is an optimal stopping time in (4).

The next important lemma gives the property of discounted stock price process. The proof can be found in [6].
Lemma 3. Suppose that $\eta>\widetilde{\eta}$ are two roots of

$$
x^{2}-\left(\mu_{1}-\lambda_{1}+\mu_{2}-\lambda_{2}\right) x+\left(\mu_{1}-\lambda_{1}\right)\left(\mu_{2}-\lambda_{2}\right)-\lambda_{1} \lambda_{2}=0,
$$

where $\lambda_{1}, \lambda_{2}$ are defined in (1). If $\eta>r$, then $e^{-r t} X_{t}$ is a submartingale. If $\eta \leq r$, then $e^{-r t} X_{t}$ is a supermartingale.

Remark 1. Under Assumption 1, a direct computation shows

$$
\eta=\frac{\mu_{1}-\lambda_{1}+\mu_{2}-\lambda_{2}+\sqrt{\Gamma}}{2}
$$

where

$$
\Gamma=\left(\mu_{1}-\lambda_{1}+\mu_{2}-\lambda_{2}\right)^{2}-4\left(\left(\mu_{1}-\lambda_{1}\right)\left(\mu_{2}-\lambda_{2}\right)-\lambda_{1} \lambda_{2}\right) .
$$

Simple calculation gives that

$$
\begin{aligned}
& \left(\mu_{2}-\mu_{1}+\lambda_{1}+\lambda_{2}\right)^{2}-\Gamma=4 \lambda_{2}\left(\mu_{2}-\mu_{1}\right)>0, \\
& \Gamma-\left(\mu_{1}+\lambda_{1}-\mu_{2}+\lambda_{2}\right)^{2}=-4 \lambda_{1}\left(\mu_{1}-\mu_{2}\right)>0,
\end{aligned}
$$

which implies that $\mu_{1}<\eta<\mu_{2}$. We will see that the root $\eta$ affects the shape of the stopping region in the following proposition and the next section. Economically speaking, the decisions to sell the stock are closely related to $\eta$.

Proposition 1. Let $\eta$ be defined by (10).

Case 1. If $\eta>r$, then the optimal stopping time $\tau_{\mathscr{D}}=T-t$, and

$$
V(t, x, i)=\mathbb{E}\left[e^{-r(T-t)} g\left(X_{T-t}^{x}\right)\right]
$$

Case 2. If $\eta \leq r$, then the function $x \longmapsto V(t, x, i)-g(x)$ is decreasing. Moreover, $\mathscr{D}$ is upward connected: if $\left(t, x^{\prime}, i\right) \in \mathscr{D}$, then $(t, x, i) \in \mathscr{D}$ for $x>x^{\prime}$.

Proof. If $\eta>r$, by Lemma 3, it is easy to see that $e^{-r t} g\left(X_{t}\right)$ is also a submartingale. By optional sampling theorem, for any $\tau \in[0, T-t]$,

$$
\mathbb{E}\left[e^{-r \tau} g\left(X_{\tau}^{x}\right)\right] \leq \mathbb{E}\left[e^{-r(T-t)} g\left(X_{T-t}^{x}\right)\right]
$$

Hence, by the definition of $V($ see $(4))$, we conclude that $\tau=T-t$ is an optimal stopping time of problem (10) and

$$
V(t, x, i)=\mathbb{E}\left[e^{-r(T-t)} g\left(X_{T-t}^{x}\right)\right] .
$$

If $\eta \leq r$, for any $x^{\prime}<x$, let $\tau$ be the optimal stopping time for $V(t, x, i)$, then $\tau$ is suboptimal for $V\left(t, x^{\prime}, i\right)$. By Lemma $3, e^{-r t} X_{t}$ is supermartingale. The optional sampling theorem leads to 


$$
\begin{aligned}
V\left(t, x^{\prime}, i\right)-V(t, x, i) & \geq \mathbb{E}\left[e^{-r \tau}\left(X_{\tau}^{\dot{x}}-a\right)\right]-\mathbb{E}\left[e^{-r \tau}\left(X_{\tau}^{x}-a\right)\right] \\
& =\mathbb{E}\left[e^{-r \tau}\left(X_{\tau}^{\dot{x}}-X_{\tau}^{x}\right)\right] \\
& =\left(x^{\prime}-x\right) \mathbb{E}\left[e^{-r \tau} \exp \left(H_{\tau}\right)\right] \\
& \geq x^{\prime}-x
\end{aligned}
$$

where $H_{t}$ is defined by

$$
H_{t}=\int_{0}^{t}\left(\mu\left(\alpha_{s}\right)-\frac{1}{2} \sigma^{2}\left(\alpha_{s}\right)\right) \mathrm{d} s+\int_{0}^{t} \sigma\left(\alpha_{s}\right) \mathrm{d} B_{s} .
$$

This implies the monotonicity of the function $x \longmapsto V(t, x, i)-g(x)$. If $\left(t, x^{\prime}, i\right) \in \mathscr{D}$, then the above inequality leads to

$$
0 \leq V(t, x, i)-g(x) \leq V\left(t, x^{\prime}, i\right)-g\left(x^{\prime}\right)=0 \text { for } x>x^{\prime} .
$$

That is, $(t, x, i) \in \mathscr{D}$.

Remark 2. From Proposition 1, we know that if the risk-free interest rate is always lower than the return rate of the stock, then it is never optimal to sell the stock before the maturity date.

\section{Main Results}

In this section, we shall investigate the shape of the stopping region of the primal problem (4). We start with some basic properties of the free boundary.
Theorem 1. Consider the problem (4) with $\mu_{1}<\eta \leq r$.

Case 1: if $\mu_{1}<\eta \leq r \leq \mu_{2}$, then there exists a continuous function $b_{1}(t)>0$ defined by

$$
b_{1}(t)=\inf \{x: V(t, x, 1)=g(x)\},
$$

such that

$$
\mathscr{D}_{1}=\left\{(t, x): x \geq b_{1}(t)\right\},
$$

and $\mathscr{D}_{2}=\varnothing$. Moreover, $b_{1}(t)$ is nonincreasing and $b_{1}(T)=r a /\left(r-\mu_{1}\right)$.

Case 2: if $\mu_{1}<\eta \leq \mu_{2}<r$, then for every $i \in \mathcal{S}$, there exists a continuous function $b_{i}(t)>0$ defined by

$$
b_{i}(t)=\inf \{x: V(t, x, i)=g(x)\},
$$

such that

$$
\mathscr{D}_{i}=\left\{(t, x): x \geq b_{i}(t)\right\} .
$$

Moreover, $b_{i}(t)$ is nonincreasing and $b_{i}(T)=r a /\left(r-\mu_{i}\right)$ for $i \in \mathcal{S}$.

Proof. Firstly, we assert that

$$
\{(t, x):-r g(x)+(\mathscr{L} g)(t, x, i)>0\} \subset \mathscr{C}_{i}, \quad i \in \mathcal{S} .
$$

By Dynkin's formula, we obtain that

$$
\mathbb{E}\left[e^{-r s} g\left(X_{s}^{x}\right)\right]=g(x)+\mathbb{E}\left[\int_{0}^{s} e^{-r v} \cdot(-r g+\mathscr{L} g)\left(t+v, X_{v}^{x}, \alpha_{v}\right) \mathrm{d} v\right] .
$$

For any $(t, x) \in\{(t, x):-r g(x)+(\mathscr{L} g)(t, x, i)>0\}$, we can choose an open neighborhood $U \subset[0, T] \times(0,+\infty)$ of $(t, x)$ such that

$$
-r g(x)+(\mathscr{L} g)(t, x, i)>0,
$$

for any $(t, x) \in U$. Let $s=\tau_{U}$ in (25) be the first exist time of $U$ when $\left(t+s, X_{s}^{x}\right)$ started at $(t, x)$ at time 0 . Denote $T_{i}$ to be the first transition time of $\alpha$ with $\alpha(0)=i$. Since $-r g\left(X_{v}^{x}\right)+$ $(\mathscr{L} g)\left(t+v, X_{v}^{x}, \alpha_{v}\right)>0$ for $v \in\left(0, \tau_{U} \wedge T_{i}\right)$ a.s., by (4), we derive that

$$
\begin{aligned}
V(t, x, i) \geq & \mathbb{E}\left[e^{-r\left(\tau_{U} \wedge T_{i}\right)} g\left(X_{\tau_{U} \wedge T_{i}}^{x}\right)\right]=g(x)+\mathbb{E}\left[\int_{0}^{\tau_{U} \wedge T_{i}} e^{-r v}\right. \\
& \left.\cdot(-r g+\mathscr{L} g)\left(t+v, X_{v}^{x}, \alpha_{v}\right) \mathrm{d} v\right] \\
> & g(x) .
\end{aligned}
$$

This implies that $(t, x) \in \mathscr{C}_{i}$.
Case 1: (24) is equivalent to

$$
\mathscr{D}_{i} \subset\{(t, x):-r g(x)+(\mathscr{L} g)(t, x, i) \leq 0\}, \quad i \in \mathcal{S} \text {. }
$$

Under the assumption that $\mu_{1}<r \leq \mu_{2}$, this leads to $\mathscr{D}_{1} \subset\left\{(t, x): x \geq r a /\left(r-\mu_{1}\right)\right\}$ and $\mathscr{D}_{2}=\varnothing$. Now, we shall follow the steps in [19] to show that the value function $V(t, x, i)$ satisfies

$$
V_{t}(t, x, i)+(\mathscr{A} V)(t, x, i)-r V(t, x, i)=0 \text { in } \mathscr{C} .
$$

Define a killed process

$$
\widetilde{X}_{t}:= \begin{cases}X_{t}, & \text { if } t<T \\ \Delta, & \text { if } t \geq T\end{cases}
$$

where $T$ is a random variable exponentially distributed with parameter $r>0$ and independent of $X, \Delta$ is a 
fictitious point, and all functions are assumed to take value zero at $\Delta$. By a direct computation, we have

$$
\begin{aligned}
\mathbb{E}\left[g\left(\tilde{X}_{t}\right)\right]= & \mathbb{E}\left[g\left(X_{t}\right) 1_{t<T}\right] \\
= & \mathbb{E}\left[g\left(X_{t}\right) 1_{t<T} \mid t<T\right] \mathbb{P}(T>t) \\
& +\mathbb{E}\left[g\left(X_{t}\right) 1_{t<T} \mid t \geq T\right] \mathbb{P}(T \leq t) \\
= & \mathbb{E}\left[e^{-r t} g\left(X_{t}\right)\right],
\end{aligned}
$$

where the second equality follows from law of total expectation and the last equality follows from the fact that $X_{t}$ is independent of $T$. Now, we can rewrite the value function as

$$
V(t, x, i)=\mathbb{E}\left[g\left(\tilde{X}_{\tau_{\mathscr{D}}}^{x}\right)\right] .
$$

We choose $(t, x, i) \in \mathscr{C}$ and a neighborhood $U \subset \mathscr{C}$ of $(t, x, i)$. Define $\sigma=\inf \{s \in[0, T-t]:(t+s$, $\left.\left.X_{s}^{x}, \alpha_{s}\right) \notin U\right\}$. It is clear $\sigma \leq \tau_{\mathscr{D}}$. This leads to $\sigma+\tau_{\mathscr{D}}{ }^{\circ} \theta_{\sigma}=\tau_{\mathscr{D}}$ (see [19]). By strong Markov property,

$$
\begin{aligned}
\mathbb{E}\left[V\left(\sigma, \tilde{X}_{\sigma}, \alpha_{\sigma}\right)\right] & =\mathbb{E}\left[\mathbb{E}\left[g\left(\tilde{X}_{\tau_{\mathscr{D}}}\right) \circ \theta_{\sigma} \mid \mathscr{F}_{\sigma}\right]\right] \\
& =\mathbb{E}\left[\mathbb{E}\left[g\left(\tilde{X}_{\sigma+\tau_{\mathscr{D}}{ }^{\circ} \theta_{\sigma}}\right) \mid \mathscr{F}_{\sigma}\right]\right] \\
& =\mathbb{E}\left[\mathbb{E}\left[g\left(\tilde{X}_{\tau_{\mathscr{D}}}\right) \mid \mathscr{F}_{\sigma}\right]\right] \\
& =\mathbb{E}\left[g\left(\tilde{X}_{\tau_{\mathscr{D}}}\right)\right] \\
& =V(t, x, i) .
\end{aligned}
$$

Here, $\theta$ is the shift operator. This implies the characteristic operator of $\left(t, \widetilde{X}_{t}, \alpha_{t}\right)$ is identically zero:

$$
\lim _{U \longrightarrow(t, x, i)} \frac{\mathbb{E}\left[V\left(\sigma, \widetilde{X}_{\sigma}, \alpha_{\sigma}\right)\right]-V(t, x, i)}{\mathbb{E}[\sigma]}=0 .
$$

By the fact that the characteristic operator coincides with the infinitesimal generator of $\left(t, \widetilde{X}_{t}, \alpha_{t}\right)$, we derive that (29) holds.

We claim that $\mathscr{D}_{1} \neq \varnothing$. Otherwise, by (29) and letting $t \longrightarrow T^{-}$, we obtain that

$$
\begin{aligned}
\lim _{t \longrightarrow T^{-}} V_{t}(t, x, 1)= & r V(T, x, 1)-\frac{1}{2} x^{2} \sigma^{2} V_{x x}(T, x, 1) \\
& -\mu_{1} x V_{x}(T, x, 1)-\lambda_{1}(V(T, x, 2) \\
& -V(T, x, 1)) \\
= & \left(r-\mu_{1}\right) x-r a>0,
\end{aligned}
$$

for $x>r a /\left(r-\mu_{1}\right)$, where the second equality follows from the fact that $V(T, x, i)=g(x)$. As $V_{t}(t, x, 1) \leq 0$ (Lemma 1), this leads to a contradiction. By Proposition 1 and $\mathscr{D}_{1} \neq \varnothing$, we obtain that $b_{1}(t)$ defined by (20) is well defined and (21) holds.

We shall deduce that $b_{1}(t)$ is nonincreasing. Otherwise, there exist $t_{1}<t_{2}<T$ such that $b_{1}\left(t_{1}\right)<b_{1}\left(t_{2}\right)$. By the definition of $b_{1}(t)$, we derive that

$$
V\left(t_{2}, b_{1}\left(t_{1}\right), 1\right)>g\left(b_{1}\left(t_{1}\right)\right)=V\left(t_{1}, b_{1}\left(t_{1}\right), 1\right) .
$$

This contradicts with the fact that $V(\cdot, x, i)$ is nonincreasing (Lemma 1).

Next, we prove the continuity of $b_{1}(t)$. Otherwise, suppose that there exist some $t_{0} \in[0, T)$ such that

$$
0 \leq x_{2}=\lim _{t \longrightarrow t_{0}^{+}} b_{1}(t)<x_{1}=\lim _{t \longrightarrow t_{0}^{-}} b_{1}(t) .
$$

Then, the domain $\mathcal{N}=\left(0, t_{0}\right) \times\left(x_{2}, x_{1}\right) \times\{1\} \subset \mathscr{C}_{1}$. For any $\epsilon>0$, by a similar argument as (33), we have

$$
\mathbb{E}\left[V\left(t+\varepsilon, \widetilde{X}_{\varepsilon}, \alpha_{\varepsilon}\right)\right]=\mathbb{E}\left[\mathbb{E}\left[g\left(\widetilde{X}_{\tau_{\mathscr{D}}}\right) \circ \theta_{\varepsilon} \mid \mathscr{F}_{\varepsilon}\right]\right]=\mathbb{E}\left[g\left(\widetilde{X}_{\tau_{\mathscr{P}}+\varepsilon}\right)\right] \leq \sup _{\tau \in \Lambda_{t, T}} \mathbb{E}\left[g\left(\widetilde{X}_{\tau}\right)\right]=V(t, x, i),
$$

where the inequality follows from the fact that $\tau_{\mathscr{D}}(t+\epsilon, x, i) \leq T-(t+\epsilon)$. By Dynkin's formula and (30), we have

$$
\begin{aligned}
\mathbb{E}\left[V\left(t+\varepsilon, \widetilde{X}_{\varepsilon}, \alpha_{\varepsilon}\right)\right]-V(t, x, i) & =\mathbb{E}\left[e^{-r \varepsilon} V\left(t+\varepsilon, X_{\varepsilon}, \alpha_{\varepsilon}\right)\right]-V(t, x, i) \\
& =\mathbb{E}\left[\int_{0}^{\varepsilon} e^{-r v}\left(V_{t}+\mathscr{A} V-r V\right)\left(t+v, X_{v}^{x}, \alpha_{v}\right) \mathrm{d} v\right] \\
& \leq 0,
\end{aligned}
$$

where the last inequality follows from (38). Dividing the above inequality by $\epsilon$, letting $\epsilon \longrightarrow 0$, and using the mean value theorem, we derive 


$$
\left(V_{t}+\mathscr{A} V-r V\right)(t, x, i) \leq 0
$$

We fix $i=1$ and let $t \longrightarrow t_{0}^{+}$. As $V\left(t_{0}+\delta, x, 1\right)=g(x)$ for $\delta>0$ and $x \in\left(x_{2}, x_{1}\right)$, by (40), we have

$$
\begin{array}{r}
r V\left(t_{0}^{+}, x, 1\right)-(\mathscr{A} V)\left(t_{0}^{+}, x, 1\right) \geq V_{t}\left(t_{0}^{+}, x, 1\right)=0, \\
\text { for } x \in\left(x_{2}, x_{1}\right) .
\end{array}
$$

$$
V\left(t_{0}, x, 2\right) \leq \frac{1}{\lambda_{1}}\left(\left(r-\mu_{1}+\lambda_{1}\right) x-\left(\lambda_{1}+r\right) a\right)
$$

$$
\text { for } x \in\left(x_{2}, x_{1}\right) \text {. }
$$

On the other hand, for any $(t, x, 1) \in \mathcal{N}$, by (29), letting $t \longrightarrow t_{0}^{-}$, we derive

That means

$$
\begin{aligned}
V\left(t_{0}, x, 2\right) & =\frac{1}{\lambda_{1}}\left(r V\left(t_{0}, x, 1\right)-\left(V_{t}+\mathscr{L} V\right)\left(t_{0}, x, 1\right)+\lambda_{1} V\left(t_{0}, x, 1\right)\right) \\
& \geq \frac{1}{\lambda_{1}}\left(\left(r-\mu_{1}+\lambda_{1}\right) x-\left(\lambda_{1}+r\right) a\right), \quad \text { for } x \in\left(x_{2}, x_{1}\right),
\end{aligned}
$$

where the last inequality follows from $V\left(t_{0}, x, 1\right)=g(x)$ for $x \in\left(x_{2}, x_{1}\right)$ and $V_{t} \leq 0$ (Lemma $1)$. By (41) and (43), as $r>\mu_{1}$, we derive that

$$
V_{x}\left(t_{0}, x, 2\right)=\frac{r-\mu_{1}+\lambda_{1}}{\lambda_{1}}>1 \text {, for } x \in\left(x_{2}, x_{1}\right) \text {, }
$$

which is in contradiction with Proposition 1. Hence, $b_{1}(\cdot)$ is continuous.

Finally, we prove $b_{1}(T)=r a /\left(r-\mu_{1}\right)$. As $b_{1}(t)$ is nonincreasing and $\mathscr{D}_{1} \subset\left\{(t, x): x \geq r a /\left(r-\mu_{1}\right)\right\}$, we have $b_{1}(t)$ is well defined and $b_{1}(T):=\lim _{t \rightarrow T^{-}} b_{1}$ $(t) \geq r a /\left(r-\mu_{1}\right):=x^{*}$. Suppose that $b_{1}(T)>x^{*}$. For any $\bar{x} \in\left(x^{*}, b_{1}(T)\right)$, since $V(T, x, i)=g(x)$, letting $t \longrightarrow T^{-}$in (29), we have

$$
\begin{aligned}
V_{t}(T, \bar{x}, 1) & =r V(T, \bar{x}, 1)-(\mathscr{A} V)(T, \bar{x}, 1) \\
& =\left(r-\mu_{1}\right) \bar{x}-r a>0,
\end{aligned}
$$

which contradicts with $V_{t} \leq 0$ (Lemma 1).

Case 2: we notice that $\mathscr{D}_{i} \subset\left\{(t, x): x \geq r a /\left(r-\mu_{i}\right)\right\}$. Now, the proof is similar to Case 1 .

In the following theorem, we rewrite the optimal stopping problem (4) as a free boundary problem.

Theorem 2. Assume that $\eta \leq r$.

Case 1: if $\mu_{1}<\eta \leq r \leq \mu_{2}$, then the value function $V$ is the unique solution to the following problem:

$$
\begin{aligned}
V_{t}(t, x, 1)+(\mathscr{A} V)(t, x, 1)-r V(t, x, 1) & =0 \text { in } \mathscr{C}_{1}=\left\{(t, x): x<b_{1}(t)\right\}, \\
V_{t}(t, x, 2)+(\mathscr{A} V)(t, x, 2)-r V(t, x, 2) & =0 \text { in }(0, T) \times(0,+\infty), \\
V\left(t, b_{1}(t), 1\right) & =g\left(b_{1}(t)\right), \quad t \in[0, T), \\
V_{x}\left(t, b_{1}(t), 1\right) & =1, \quad t \in[0, T), \\
V(T, x, i) & =g(x), \quad x \in(0,+\infty), i \in \mathcal{S} .
\end{aligned}
$$

In particular, the partial derivatives $V_{x x}, V_{x}$, and $V_{t}$ exist and are continuous in $\mathscr{C}$.
Case 2: if $\mu_{1}<\eta \leq \mu_{2}<r$, then the value function $V$ defined in (4) is the unique solution to the following free boundary problem:

$$
\begin{aligned}
V_{t}(t, x, i)+(\mathscr{A} V)(t, x, i)-r V(t, x, i) & =0 \text { in } \mathscr{C}=\left\{(t, x, i): x<b_{i}(t)\right\}, \\
V\left(t, b_{i}(t), i\right) & =g\left(b_{i}(t)\right), \quad t \in[0, T), i \in \mathcal{S}, \\
V_{x}\left(t, b_{i}(t), i\right) & =1, \quad t \in[0, T), i \in \mathcal{S}, \\
V(T, x, i) & =g(x), \quad x \in[0+\infty), i \in \mathcal{S} .
\end{aligned}
$$


In particular, the partial derivatives $V_{x x}, V_{x}$, and $V_{t}$ exist and are continuous in $\mathscr{C}$.

Proof. We only prove Case 2 . Case 1 can be proved similarly. (47) follows directly from (29). (48) follows from the continuity of $V$ (Lemma 2). (50) follows from (4) directly.

We shall prove the smooth pasting condition (49). We set $x=b_{i}(t)$. As $(t, x-\epsilon) \in \mathscr{C}_{i}$, we derive that

$$
\frac{V(t, x, i)-V(t, x-\epsilon, i)}{\epsilon} \leq \frac{x-a-(x-\epsilon-a)}{\epsilon}=1,
$$

where the inequality follows from (48). Let $\epsilon \longrightarrow 0$,

$$
V_{x}^{-}(t, x, i) \leq 1,
$$

where $V_{x}^{-}$is the left derivative of $V$ in $x$. For any $\epsilon>0$, let $\tau_{\epsilon}^{\prime}$ be the optimal stopping time for $V(t, x-\epsilon, i)$ and $T_{i}$ be the first transition time of $\alpha$ with $\alpha(0)=i$. Denote

$$
\tau_{\varepsilon}:=\inf \left\{s \in\left[0, T_{i}\right] \mid X_{s}^{x-\varepsilon} \geq b_{i}(t+s)\right\} .
$$

By (9) and Theorem 1 , if $T_{i} \geq T-t$, then $\tau_{\epsilon}^{\prime}=\tau_{\epsilon}$; otherwise, we have

$$
\begin{aligned}
\tau_{\epsilon}^{\prime}(t, x, i) & =\inf \left\{s \in[0, T-t] \mid X_{s}^{x-\epsilon} \geq b_{\alpha_{s}}(t+s)\right\} \\
& =\tau_{\epsilon} \wedge \inf \left\{s \in\left[T_{i}, T-t\right] \mid X_{s}^{x-\epsilon} \geq b_{\alpha_{s}}(t+s)\right\} .
\end{aligned}
$$

By the solution of $\operatorname{SDE}(2)$, we have

$$
\begin{aligned}
\tau_{\epsilon} & =\inf \left\{s \in\left[0, T_{i}\right]: \int_{0}^{s}\left(\mu\left(\alpha_{v}\right)-\frac{1}{2} \sigma^{2}\left(\alpha_{v}\right)\right) \mathrm{d} v+\int_{0}^{s} \sigma\left(\alpha_{v}\right) \mathrm{d} B_{v} \geq \log \left(\frac{b_{i}(t+s)}{x-\epsilon}\right)\right\} \\
& \leq \inf \left\{s \in\left[0, T_{i}\right]:\left(\mu_{1}-\frac{1}{2} \sigma_{1}^{2}\right) s+\sigma_{1} B_{s} \geq \log \left(\frac{x}{x-\epsilon}\right)\right\} \\
& \leq \inf \left\{s \in\left[0, T_{i}\right]:-C s+B_{s} \geq \frac{1}{\sigma_{1}} \log \left(\frac{x}{x-\epsilon}\right)\right\},
\end{aligned}
$$

where the first inequality following from $b_{i}$ is nonincreasing and the last inequality follows from the fact that $1 / \sigma_{1}\left(\mu_{1}-\right.$ $\left.(1 / 2) \sigma_{1}^{2}\right)$ can be bounded below by some constant $-C<0$.

By the law of iterated logarithm, we have for any $\epsilon \prime>0$, there exists some $s \in(0, \epsilon \prime]$ such that $\widetilde{B}_{s}>C s$ a.s. This implies that there exists some $\epsilon>0$ small enough such that

$$
\widetilde{B}_{s}-C s>\frac{1}{\sigma_{1}} \log \frac{x}{x-\varepsilon} \text { a.s. }
$$

Hence, by the definition of $\tau_{\epsilon}$, we have $\tau_{\epsilon} \leq \epsilon \prime \longrightarrow 0$, as $\epsilon \prime \longrightarrow 0$. Equation (53) leads to $\lim _{\epsilon \longrightarrow 0} \tau_{\epsilon}^{\prime}=0$.

Now, we prepare to prove $V_{x}^{-}(t, x, i) \geq 1$. Since

$$
\begin{aligned}
\frac{V(t, x, i)-V(t, x-\epsilon, i)}{\epsilon} \geq & \frac{\mathbb{E}\left[e^{-r \tau_{\epsilon}^{\prime}}\left(X_{\tau_{\epsilon}^{\prime}}^{x}-a\right)\right]-\mathbb{E}\left[e^{-r \tau_{\epsilon}^{\prime}}\left(X_{\tau_{\epsilon}^{\prime}}^{x-\epsilon}-a\right)\right]}{\epsilon} \\
& =\frac{\mathbb{E}\left[e^{-r \tau_{\epsilon}^{\prime}}\left(X_{\tau_{\epsilon}^{\prime}}^{x}-X_{\tau_{\epsilon}^{\prime}}^{x-\epsilon}\right)\right]}{\epsilon} \\
& =\mathbb{E}\left[\exp \left(H_{\tau_{\epsilon}^{\prime}}-r \tau_{\epsilon}^{\prime}\right)\right]
\end{aligned}
$$

where $H_{t}$ is defined in (18). Letting $\epsilon \longrightarrow 0$, by Fatou's lemma, we conclude that $V_{x}^{-}(t, x, i)=1$. As $V(t, x, i)=g(x)$ for $x>b_{i}(t)$, it is easy to see that $V_{x}^{+}(t, x, i)=1$. Here, $V_{x}^{+}$is the right derivative of $V$ in $x$. We conclude that the smooth pasting condition (49) holds.

For uniqueness, let $f(t, x, i)$ defined on $\overline{\mathscr{C}}$ be a solution of the free boundary problem (47). We define $M_{s}:=$ $e^{-r s} f\left(s+t, X_{s}, \alpha_{s}\right)$ and set $\tau_{\mathscr{D}}=\inf \left\{s \in[0, T-t]: X_{s}^{x} \geq\right.$ $\left.b_{\alpha_{s}}(s+t)\right\}$. By It ô's lemma,

$$
\begin{aligned}
M_{s \wedge \tau_{\mathscr{D}}}= & f(t, x, i)+\int_{0}^{s \wedge \tau_{\mathscr{D}}} e^{-r v}\left(f_{t}+\mathscr{A} f-r f\right) \\
& \cdot\left(t+v, X_{v}, \alpha_{v}\right) \mathrm{d} v+N_{s \wedge \tau_{\mathscr{D}}}
\end{aligned}
$$

where $\left\{N_{s \wedge \tau_{D}}, s \geq 0\right\}$ is a martingale. Hence, by (47), $\left\{M_{s \wedge \tau_{D}}, s \geq 0\right\}$ is a martingale. We know from the optional sampling theorem that

$$
\begin{aligned}
f( & t, x, i)=M_{0}=\mathbb{E}\left[M_{\tau_{\mathscr{D}}}\right] \\
& =\mathbb{E}\left[e^{-r \tau_{\mathscr{D}}} f\left(\tau_{\mathscr{D}}+t, X_{\tau_{\mathscr{D}}}, \alpha_{\tau_{\mathscr{D}}}\right)\right] \\
& =\mathbb{E}\left[e^{-r \tau_{\mathscr{D}}} g\left(X_{\tau_{\mathscr{D}}}\right)\right] \\
& =V(t, x, i),
\end{aligned}
$$

where the second equality follows from that $\left\{M_{s \wedge \tau_{D}}, s \geq 0\right\}$ is a bounded martingale and dominated convergent theorem, the fourth equality follows from (48) and definition of $\tau_{\mathscr{D}}$, 
and the last equality follows from that $\tau_{\mathscr{D}}$ is an optimal stopping time of $V(t, x, i)$. This implies the uniqueness.

By Lemma 2 and regularity of parabolic equation (see [20]), it is obvious that $V_{t}, V_{x}, V_{x x}$ exist and are continuous in $\mathscr{C}$.

In the following, we decompose the value function into two components and derive a Volterra type integral equation. Firstly, we introduce the following notations:

$$
\begin{aligned}
& J_{i}(t, x):= \mathbb{E}\left[e^{-\left(r+\lambda_{i}\right)(T-t)} g\left(X_{T-t}^{(i)}\right)\right], \\
& K_{i}\left(t, x, u, b_{i}(u+t)\right):= \mathbb{E}\left[e^{-\left(r+\lambda_{i}\right) u} \Phi_{i}\left(X_{u}^{(i)}\right) 1_{\left\{X_{u}^{(i)}>b_{i}(u+t)\right\}}\right) \\
&\left.\left.-\lambda_{i} V\left(t+u, X_{u}^{(i)}, j\right) 1_{\left\{X_{u}^{(i)}<b_{i}(u+t)\right\}}\right)\right], \\
& j \neq i,
\end{aligned}
$$

where

$$
\Phi_{i}(x)=\left(r+\lambda_{i}-\mu_{i}\right) x+\left(r+\lambda_{i}\right) a .
$$

$X_{t}^{(i)}$ satisfies

$$
\mathrm{d} X_{t}^{(i)}=X_{t}^{(i)}\left(\mu_{i} \mathrm{~d} t+\sigma_{i} \mathrm{~d} B_{t}\right)
$$

with initial condition $X_{0}^{(i)}=x$.

Theorem 3. Assume that $\eta \leq r$.

Case 1: if $\mu_{1}<\eta \leq r \leq \mu_{2}$, then the value function defined in (4) is given by

$$
\begin{aligned}
& V(t, x, 1)=J_{1}(t, x)-\int_{0}^{T-t} K_{1}\left(t, x, u, b_{1}(u+t)\right) \mathrm{d} u \\
& \left.V(t, x, 2)=J_{2}(t, x)+\lambda_{2} \mathbb{E}\left[\int_{0}^{T-t} e^{-\left(r+\lambda_{2}\right) u} V\left(t+u, X_{u}^{(2)}, 1\right)\right) \mathrm{d} u\right]
\end{aligned}
$$

Furthermore, the free boundary $b_{1}(t)$ satisfies the following nonlinear Volterra type integral equation:

$$
g\left(b_{1}(t)\right)=J_{1}\left(t, b_{1}(t)\right)-\int_{0}^{T-t} K_{1}\left(t, b_{1}(t), u, b_{1}(u+t)\right) \mathrm{d} u .
$$

Case 2: if $\mu_{1}<\eta \leq \mu_{2}<r$, then the value function defined in (4) is given by

$$
V(t, x, i)=J_{i}(t, x)-\int_{0}^{T-t} K_{i}\left(t, x, u, b_{i}(u+t)\right) \mathrm{d} u, \quad i \in \mathcal{S} .
$$

Furthermore, the free boundary $b_{i}(t)$ satisfies the following nonlinear Volterra integral equation:

$$
g\left(b_{i}(t)\right)=J_{i}\left(t, b_{i}(t)\right)-\int_{0}^{T-t} K_{i}\left(t, b_{i}(t), u, b_{i}(u+t)\right) \mathrm{d} u, \quad i \in \mathcal{S} .
$$

Proof. We only prove Case 2 and Case 1 can be proved by a similar method. Firstly, we consider the following optimal stopping problem:

$$
V_{i}(t, x)=\sup _{0 \leq \tau \leq T-t} \mathbb{E}\left[e^{-\left(r+\lambda_{i}\right) \tau} g\left(X_{\tau}^{(i)}\right)+\lambda_{i} \int_{0}^{\tau} e^{-\left(r+\lambda_{i}\right) u} V\left(t+u, X_{u}^{(i)}, j\right)\right], \quad j \neq i
$$

where $V(t, x, i)$ is defined in (4) and $X_{t}^{(i)}$ satisfies (63). Fixing $i$ in Case 2 of Theorem 2, by a similar Markovian argument, we conclude that $V_{i}(t, x)$ is a solution to the free boundary problem (47)-(50). The uniqueness implies that $V_{i}(t, x)=V(t, x, i)$ for $(t, x, i) \in[0, T] \times(0,+\infty) \times \mathcal{S}$.

By the regularity of $V$ in Case 2 of Theorem 2, we can use the local time-space formula given in [19] to obtain that

$$
\begin{aligned}
e^{-\left(r+\lambda_{i}\right) s} V_{i}\left(s+t, X_{s}^{(i)}\right)= & V_{i}(t, x)+\int_{0}^{s} e^{-\left(r+\lambda_{i}\right) s}\left(V_{i t}+\frac{1}{2} \sigma_{i}^{2} x^{2} V_{i x x}\right. \\
& \left.+\mu_{i} x V_{i x}-\left(r+\lambda_{i}\right) V_{i}\right)\left(t+u, X_{u}^{(i)}\right) 1_{\left\{X_{u}^{(i)} \neq b_{i}(u+t)\right\}} \mathrm{d} u \\
& +\int_{0}^{s} \sigma_{i} X_{u}^{(i)} V_{i x}\left(t+u, X_{u}^{(i)}\right) 1_{\left\{X_{u}^{(i)} \neq b_{i}(u+t)\right\}} \mathrm{d} B_{u} \\
& +\frac{1}{2} \int_{0}^{s}\left(V_{i x}\left(t+u, X_{u}^{(i)}+\right)-V_{i x}\left(t+u, X_{u}^{(i)}-\right)\right) \\
& \cdot \mathbb{1}_{\left\{X_{u}^{(i)}=b_{i}(u+t)\right\}} d e_{u}^{b_{i}}\left(X^{(i)}\right),
\end{aligned}
$$


where $\ell_{u}^{b_{i}}\left(X^{(i)}\right)$ is the local time of $X^{(i)}$ at the curve $b_{i}$ given by

$$
\ell_{u}^{b_{i}}\left(X^{(i)}\right):=\lim _{\epsilon \longrightarrow 0} \frac{1}{2 \epsilon} \int_{0}^{u} 1_{\left\{b_{i}(v)-\epsilon<X_{v}^{(i)}<b_{i}(v)+\epsilon\right\}} d<X^{(i)}>{ }_{v},
$$

and $\mathrm{d} \ell_{u}^{b_{i}}\left(X^{(i)}\right)$ refers to integration with respect to the continuous increasing function $u \mapsto \ell_{u}^{b_{i}}\left(X^{(i)}\right)$.

We notice that $V_{i}(t, x)=g(x)$ for $x>b_{i}(t)$ and $V_{i}$ satisfies (47) for $x<b_{i}(t)$. Hence,

$$
\left(V_{i t}+\frac{1}{2} \sigma_{i}^{2} x^{2} V_{i x x}+\mu_{i} x V_{i x}-\left(r+\lambda_{i}\right) V_{i}\right)\left(t+u, X_{u}^{(i)}\right)= \begin{cases}\Phi_{i}\left(X_{u}^{(i)}\right), & \text { if } X_{u}^{(i)}>b_{i}(u+t), \\ \lambda_{i} V\left(u, X_{u}^{(i)}, j\right), & \text { if } X_{u}^{(i)}<b_{i}(u+t) .\end{cases}
$$

Here, $j \neq i$. Setting $s=T-t$ together with (49) and (50), taking expectation in (69), and using the notation (60) and (61), we derive that

$$
\begin{aligned}
V_{i}(t, x)=\mathbb{E} & {\left[e^{-\left(r+\lambda_{i}\right)(T-t)} g\left(X_{T-t}\right)\right]-\mathbb{E}\left[\int_{0}^{T-t} e^{-\left(r+\lambda_{i}\right) u}\left[\Phi_{i}\left(X_{u}^{(i)}\right) 1_{\left\{X_{u}^{(i)}>b_{i}(u+t)\right\}}\right)\right.} \\
& \left.\left.-\lambda_{i} V\left(t+u, X_{u}^{(i)}, j\right) 1_{\left\{X_{u}^{(i)}<b_{i}(u+t)\right\}}\right) \mathrm{d} u\right] \\
= & J_{i}(t, x)-\int_{0}^{T-t} K_{i}\left(t, x, u, b_{i}(u+t)\right) \mathrm{d} u \\
= & V(t, x, i) .
\end{aligned}
$$

Finally, by condition (48), we derive that

$$
V_{i}^{0}(t, x)=\sup _{0 \leq \tau \leq T-t} \mathbb{E}\left[e^{-r \tau} g\left(X_{\tau}^{(i)}\right)\right], \quad i \in \mathcal{S} .
$$

$J_{i}\left(t, b_{i}(t)\right)-\int_{0}^{T-t} K_{i}\left(t, b_{i}(t), u, b_{i}(u+t)\right) \mathrm{d} u=g\left(b_{i}(t)\right)$,

$i \in \mathcal{S}$.

The next result compares the optimal selling strategies in different states.

Theorem 4. Suppose Assumption 1 holds, $\sigma_{1}<\sigma_{2}$, and $r>\mu_{2}$. Let $X_{t}^{(i)}$ be defined as (63). Then,

$$
b_{1}^{0}(t) \leq b_{1}(t) \leq b_{2}(t) \leq b_{2}^{0}(t), \quad 0<t<T,
$$

where $b_{i}^{0}(i \in \mathcal{S})$ is the free boundary of the following optimal stopping problem
Proof. We set $V(t, x, i):=V_{i}(t, x)$ for $i \in \mathcal{S}$ and $\mathcal{N}:=\left\{(t, x): V_{2}(t, x)<V_{1}(t, x)\right\}$. We shall prove $\mathcal{N}=\varnothing$. Otherwise, in $\mathscr{N}$,

$$
V_{1}(t, x)>V_{2}(t, x) \geq g(x),
$$

$$
\begin{aligned}
& V_{1 t}+\frac{1}{2} \sigma_{2}^{1} x^{2} V_{1 x x}+\mu_{1} x V_{1 x}-r V_{1}=-\lambda_{1}\left(V_{2}-V_{1}\right) \geq 0, \\
& V_{2 t}+\frac{1}{2} \sigma_{2}^{2} x^{2} V_{2 x x}+\mu_{2} x V_{2 x}-r V_{2} \leq-\lambda_{2}\left(V_{1}-V_{2}\right) \leq 0,
\end{aligned}
$$

where the last two inequalities follow from (29) and (40). By Lemma 1, it follows that

$$
\begin{aligned}
V_{2 t} & +\frac{1}{2} \sigma_{1}^{2} x^{2} V_{2 x x}+\mu_{1} x V_{2 x}-r V_{2} \\
& =V_{2 t}+\frac{1}{2} \sigma_{2}^{2} x^{2} V_{2 x x}+\mu_{2} x V_{2 x}-r V_{2}+\frac{1}{2} x^{2}\left(\sigma_{1}^{2}-\sigma_{2}^{2}\right) V_{2 x x}+\left(\mu_{1}-\mu_{2}\right) x V_{2 x}
\end{aligned}
$$


Furthermore, $V_{1}(T, x)=V_{2}(T, x)$ and $V_{1}=V_{2}$ on the boundary of $\mathcal{N}$. Hence, the comparison principle of variational inequality implies that $V_{2} \geq V_{1}$ in $\mathcal{N}$, which is a contradiction with definition of $\mathcal{N}$. This leads to $V_{2} \geq V_{1}$ for $(t, x) \in(0, T) \times(0,+\infty)$. That means $\mathscr{C}_{1} \subset \mathscr{C}_{2}$, i.e., $b_{1}(t) \leq b_{2}(t)$.

By Markovian argument, $V_{i}^{0}$ satisfies

$$
\begin{aligned}
\min \left\{-V_{i t}^{0}-\frac{1}{2} \sigma_{i}^{2} x^{2} V_{i x x}^{0}-\mu_{i} x V_{i x}^{0}+r V_{i}^{0}, V_{i}^{0}-g(x)\right\} & =0, \\
V_{i}^{0}(T, x) & =g(x) .
\end{aligned}
$$

The above inequality implies that

$$
\begin{aligned}
& -V_{2 t}^{0}-\frac{1}{2} \sigma_{2}^{2} x^{2} V_{2 x x}^{0}-\mu_{2} x V_{2 x}^{0}+r V_{2}^{0}-\lambda_{1}\left(V_{1}-V_{2}\right) \\
& \geq-\lambda_{2}\left(V_{1}-V_{2}\right) \geq 0,
\end{aligned}
$$

and by (40),

$$
-V_{1 t}-\frac{1}{2} \sigma_{1}^{2} x^{2} V_{1 x x}-\mu_{1} x V_{1 x}+r V_{1} \geq \lambda_{1}\left(V_{2}-V_{1}\right) \geq 0 .
$$

Applying comparison principle of variational inequality, we deduce that $V_{1}^{0} \leq V_{1}$ and $V_{2} \leq V_{2}^{0}$, i.e., $b_{1}^{0}(t) \leq b_{1}(t)$ and $b_{2}(t) \leq b_{2}^{0}(t)$.

In the end of this section, we perform some sensitivity analyses of the optimal selling strategy.

Theorem 5. Assume that $r \geq \eta$. The free boundary $b_{i}(t)$ of problem (4) is increasing with respect to $\mu_{i}, \sigma_{i}(i \in \mathcal{S})$, and a and decreasing with respect to $r$.

Proof. Assume that $\left(\widehat{V}_{1}, \widehat{V}_{2}\right)$ is the solution to variational inequality

$$
\min \left\{\left(r V-V_{t}-\mathscr{A} V\right)(t, x, i), V(t, x, i)-g(x)\right\}=0,
$$

for any $(t, x, i) \in(0, T) \times(0,+\infty) \times \mathcal{S}$, where $\sigma_{i}$ is $\widehat{\sigma}_{i}$, $\left(\bar{V}_{1}, \bar{V}_{2}\right)$ is the solution to variational inequality $(81)$, where $\sigma_{i}$ is $\bar{\sigma}_{i}$, and $\widehat{\sigma}_{i}>\bar{\sigma}_{i}$ for each $i \in \mathcal{S}$. By Lemma 1 , it follows that

$$
\begin{aligned}
\widehat{V}_{i t} & +\frac{1}{2} \bar{\sigma}_{i}^{2} x^{2} \widehat{V}_{i x x}+\mu_{i} x \widehat{V}_{i x}-r \widehat{V}_{i}+\lambda_{i}\left(\widehat{V}_{j}-\widehat{V}_{i}\right) \\
& =\frac{1}{2} x^{2}\left(\bar{\sigma}_{i}^{2}-\widehat{\sigma}_{i}^{2}\right) \widehat{V}_{i x x} \leq 0,
\end{aligned}
$$

if $\widehat{V}_{i}(t, x)>g(x)$, and

$$
\begin{aligned}
& \widehat{V}_{i t}+\frac{1}{2} \bar{\sigma}_{i}^{2} x^{2} \widehat{V}_{i x x}+\mu_{i} x \widehat{V}_{i x}-r \widehat{V}_{i}+\lambda_{i}\left(\widehat{V}_{j}-\widehat{V}_{i}\right) \\
& \quad \leq \frac{1}{2} x^{2}\left(\bar{\sigma}_{i}^{2}-\widehat{\sigma}_{i}^{2}\right) \widehat{V}_{i x x} \leq 0,
\end{aligned}
$$

if $\widehat{V}_{i}(t, x)=g(x)$. Employing comparison principle of variational system (see [16]), we conclude that $\widehat{V}_{i} \geq \bar{V}_{i}$, i.e., $\bar{b}_{i}(t) \leq \widehat{b}_{i}(t)$, for $i \in \mathcal{S}$. Hence, $\left(b_{1}(t), b_{2}(t)\right)$ is increasing with respect to $\sigma_{i}$ for each $i \in \mathcal{S}$.

Next, assume that $\left(\widehat{V}_{1}, \widehat{V}_{2}\right)$ is the solution to variational inequality (81), where $a$ is $a_{1},\left(\bar{V}_{1}, \bar{V}_{2}\right)$ is the solution to variational inequality (81), where $a$ is $a_{2}$, and $a_{1}<a_{2}$. Then, we have

$$
\begin{aligned}
\bar{V}_{i}(t, x) & =\sup _{0 \leq \tau \leq T-t} \mathbb{E}\left[e^{-r \tau}\left(X_{\tau}-a_{2}\right)\right] \\
& \geq a_{1}-a_{2}+\sup _{0 \leq \tau \leq T-t} \mathbb{E}\left[e^{-r \tau}\left(X_{\tau}-a_{1}\right)\right] \\
& =a_{1}-a_{2}+\widehat{V}_{i}(t, x) .
\end{aligned}
$$

This implies that if $\widehat{V}_{i}(t, x)>x-a_{1}$, then $\bar{V}_{i}(t, x) \geq \widehat{V}_{i}(t, x)+a_{1}-a_{2}>x-a_{2}$. If we denote $\hat{\mathscr{C}}_{i}$ to be the stopping region with respect to $a_{1}$ and $\overline{\mathscr{C}}_{i}$ the stopping region with respect to $a_{2}$, then $\hat{\mathscr{C}}_{i} \subset \mathscr{C}_{i}$, i.e., $\vec{b}_{i} \geq \widehat{b}_{i}$. Hence, $\left(b_{1}(t), b_{2}(t)\right)$ is increasing with respect to $a$.

In the following, assume that $\left(\widehat{V}_{1}, \widehat{V}_{2}\right)$ is the solution to variational inequality $(81)$, where $\mu_{i}$ is $\widehat{\mu}_{i},\left(\bar{V}_{1}, \bar{V}_{2}\right)$ is the solution to variational inequality (81), where $\mu_{i}$ is $\bar{\mu}_{i}$, and $\widehat{\mu}_{i}>\bar{\mu}_{i}$ for each $i \in \mathcal{S}$. By Lemma 1, it follows that

$$
\widehat{V}_{i t}+\frac{1}{2} \sigma_{i}^{2} x^{2} \widehat{V}_{i x x}+\bar{\mu}_{i} x \widehat{V}_{i x}-r \widehat{V}_{i}+\lambda_{i}\left(\widehat{V}_{j}-\widehat{V}_{i}\right)=\left(\bar{\mu}_{i}-\widehat{\mu}_{i}\right) x \widehat{V}_{i x} \leq 0,
$$

if $\widehat{V}_{i}(t, x)>g(x)$, and

$$
\widehat{V}_{i t}+\frac{1}{2} \bar{\sigma}_{i}^{2} x^{2} \widehat{V}_{i x x}+\bar{\mu}_{i} x \widehat{V}_{i x}-r \widehat{V}_{i}+\lambda_{i}\left(\widehat{V}_{j}-\widehat{V}_{i}\right) \leq\left(\bar{\mu}_{i}-\widehat{\mu}_{i}\right) x \widehat{V}_{i x} \leq 0,
$$

if $\widehat{V}_{i}(t, x)=g(x)$. Employing comparison principle of variational system (see [16]), we conclude that $\widehat{V}_{i} \geq \bar{V}_{i}$, i.e., $\bar{b}_{i}(t) \leq \widehat{b}_{i}(t)$, for $i \in \mathcal{S}$.

If we set

$$
V(t, x, i ; r)=\sup _{0 \leq \tau \leq T-t} \mathbb{E}\left[e^{-r \tau} g\left(X_{\tau}\right)\right],
$$

then for any $r_{1}<r_{2}$, it is easy to see that

$$
\begin{aligned}
V\left(t, x, i ; r_{1}\right) & =\sup _{0 \leq \tau \leq T-t} \mathbb{E}\left[e^{-r_{1} \tau} g\left(X_{\tau}\right)\right] \\
& \geq \sup _{0 \leq \tau \leq T-t} \mathbb{E}\left[e^{-r_{2} \tau} g\left(X_{\tau}\right)\right] \\
& =V\left(t, x, i ; r_{2}\right),
\end{aligned}
$$

which implies that $b_{i}(t)$ is decreasing with respect to $r$.

\section{Numerical Examples}

In this section, we present some numerical results obtained from trinomial tree method, which was put forward in [17] for pricing American option. Firstly, we briefly explain how to use the trinomial tree method to solve our problem. 

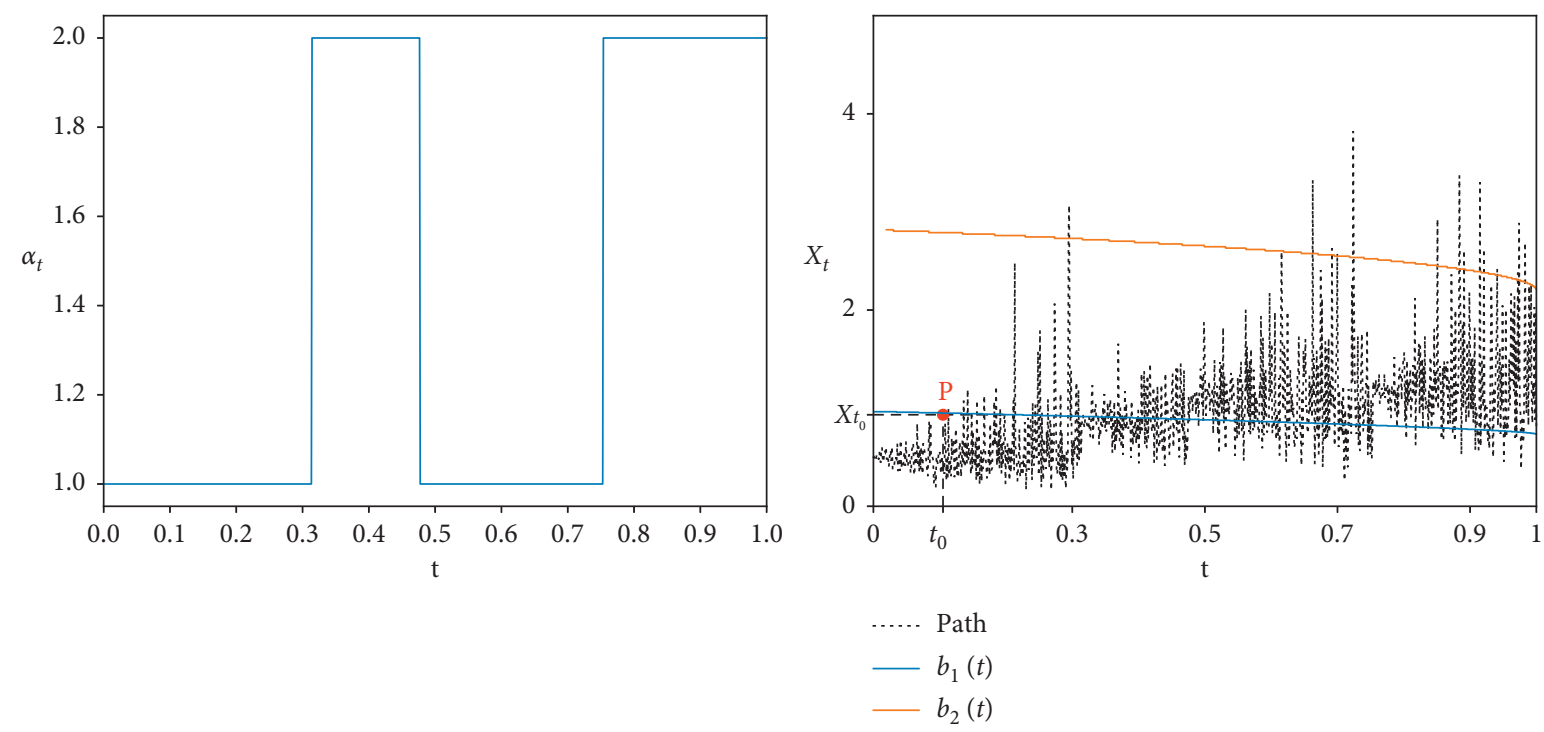

(a)
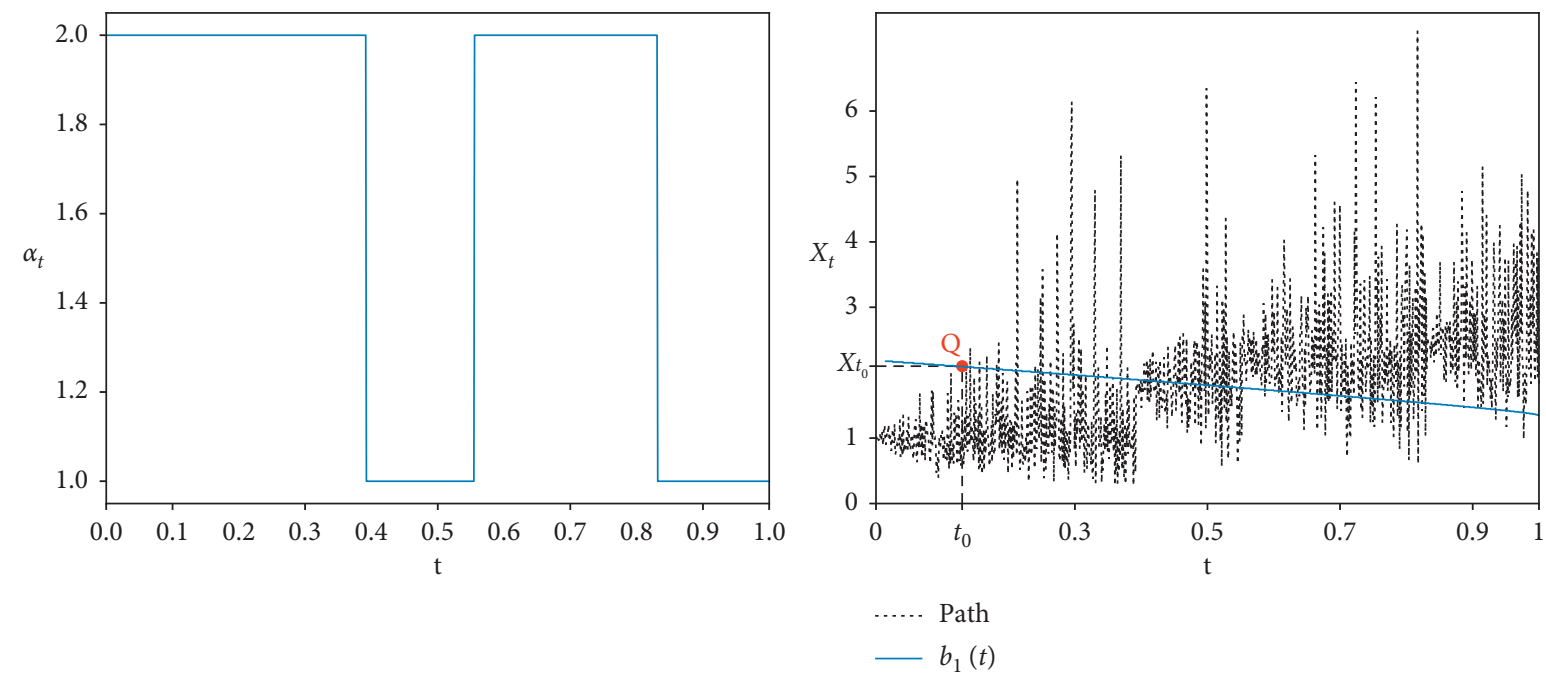

(b)

FIGURE 1: Sample path of Markov chain and stock price with (A.) $\alpha_{0}=1, S_{0}=0.5$, and early exercise boundary when $r>\mu_{2}$ and (B.) $\alpha_{0}=2$, $S_{0}=1$, and early exercise boundary when $\eta \leq r \leq \mu_{2}$.

Let $\Delta t=T / n$ be the time step-size. We take the jump ratios of the lattice as

$$
\begin{gathered}
u=e^{\sigma \sqrt{\Delta t}}, \\
d=e^{-\sigma \sqrt{\Delta t}},
\end{gathered}
$$

where

$$
\sigma=\max _{i \in \mathcal{S}}\left\{\sigma_{i}\right\}+(\sqrt{1.5}-1) \tilde{\sigma}
$$

where $\tilde{\sigma}$ is the arithmetic mean or geometric mean of $\sigma_{i}, i \in \mathcal{S}$. For regime $i$, let $\pi_{u}^{i}, \pi_{m}^{i}$, and $\pi_{d}^{i}$ be the risk neutral probabilities corresponding to the stock price increases, remaining the same, and decreases, respectively. By moment matching technique, the values of the probabilities are given by

$$
\begin{aligned}
& \pi_{m}^{i}=1-\frac{\sigma_{i}^{2}}{\sigma^{2}}, \\
& \pi_{u}^{i}=\frac{e^{\mu_{i} \Delta t}-e^{-\sigma \sqrt{\Delta t}}-\left(1-\left(\sigma^{2} / \sigma_{i}^{2}\right)\right)\left(1-e^{-\sigma \sqrt{\Delta t}}\right)}{e^{\sigma \sqrt{\Delta t}}-e^{-\sigma \sqrt{\Delta t}}},
\end{aligned}
$$

$$
\pi_{d}^{i}=\frac{e^{\sigma \sqrt{\Delta t}}-e^{\mu_{i} \Delta t}-\left(1-\left(\sigma^{2} / \sigma_{i}^{2}\right)\right)\left(e^{\sigma \sqrt{\Delta t}}-1\right)}{e^{\sigma \sqrt{\Delta t}}-e^{-\sigma \sqrt{\Delta t}}} .
$$

Let $x_{j+1}=u x_{j}$ and $x_{j-1}=d x_{j}$ and $V^{k}\left(x_{j}, i\right)$ be the trinomial approximation of the primal value function for regime $i$ at asset price $x_{j}$ and time $t_{k}=k \Delta t$. Then, the trinomial formula of the primal value function is given by, for $k=0,1, \ldots, n-1$, 


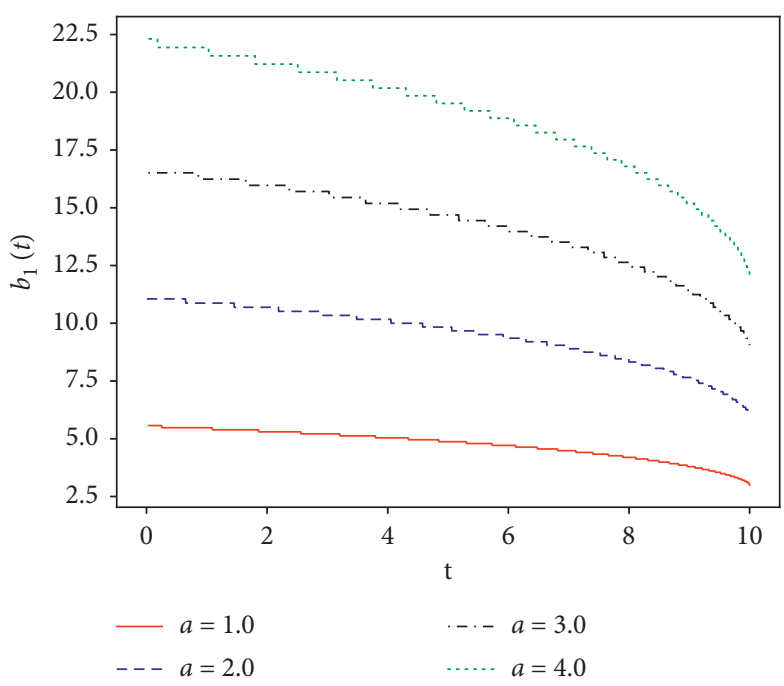

(a)

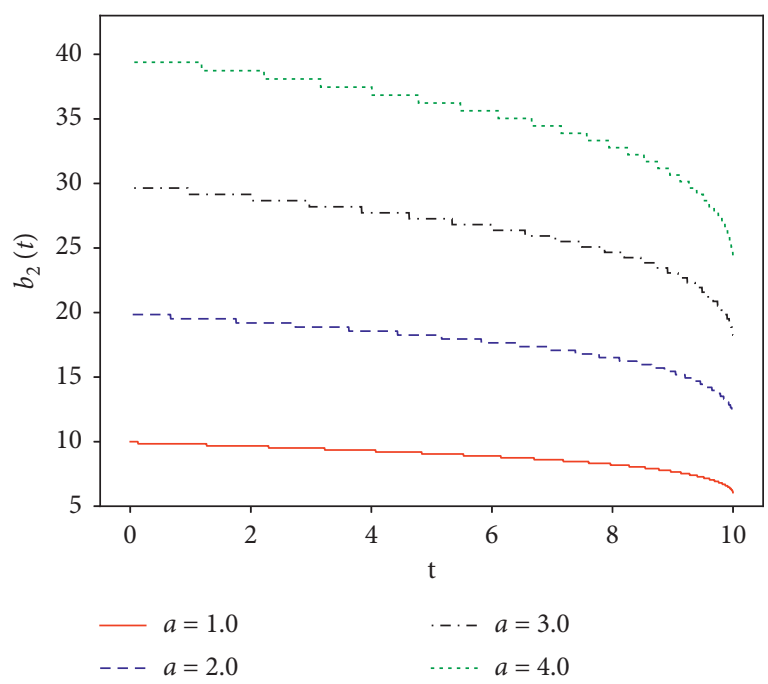

(b)

FIGURE 2: The impact of varying transaction fee on the early exercise boundary for (A.) regime 1 and (B.) regime 2.

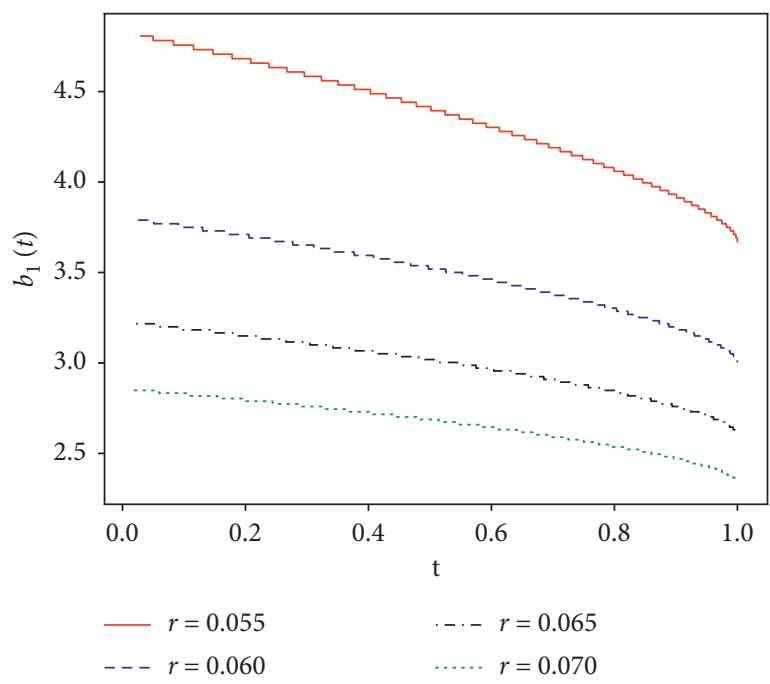

(a)

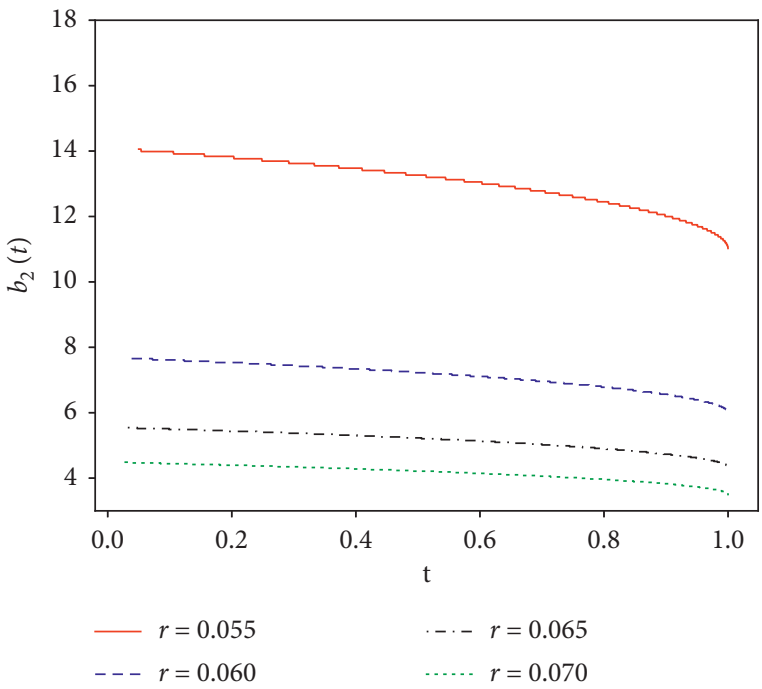

(b)

FIGURE 3: The impact of varying interest rate on the early exercise boundary for (A.) regime 1 and (B.) regime 2.

$$
\begin{aligned}
& V^{k}\left(x_{j}, i\right) \\
& \quad=\max \left\{e^{-r \Delta t} \sum_{l=1}^{2} p_{i l}\left(\pi_{u}^{i} V^{k+1}\left(x_{j+1}, l\right)+\pi_{m}^{i} V^{k+1}\left(x_{j}, l\right)+\pi_{d}^{i} V^{k+1}\left(x_{j-1}, l\right)\right), g\left(x_{j}\right)\right\},
\end{aligned}
$$

with $V^{n}\left(x_{j}, i\right)=g\left(x_{j}\right)$ for $j=0, \pm 1, \ldots, \pm n$, in which $p_{i l}$ is the transition probability from state $i$ to state $l$ for the time interval with length $\Delta t$. It is given by

$$
\left(p_{i l}\right)_{i, l \in \mathcal{S}}=e^{A \Delta t}=I+\sum_{l=1}^{\infty}(\Delta t)^{l} \frac{A^{l}}{l !},
$$

where $I$ is the identity matrix and $A$ is the generator matrix of the Markov chain process given by (1).
Example 1. In this example, the numerical experiments shed light on the optimal selling strategy of problem (4) with fixed parameters for two different cases.

The generator of Markov chain $\alpha_{t}$ is taken as

$$
A=\left(\begin{array}{cc}
-0.4 & 0.4 \\
0.5 & -0.5
\end{array}\right) \text {. }
$$

The returns of the stock are $\mu_{1}=0.04$ and $\mu_{2}=0.05$ and the volatilities $\sigma_{1}=0.25$ and $\sigma_{2}=0.45$. The maturity date is 


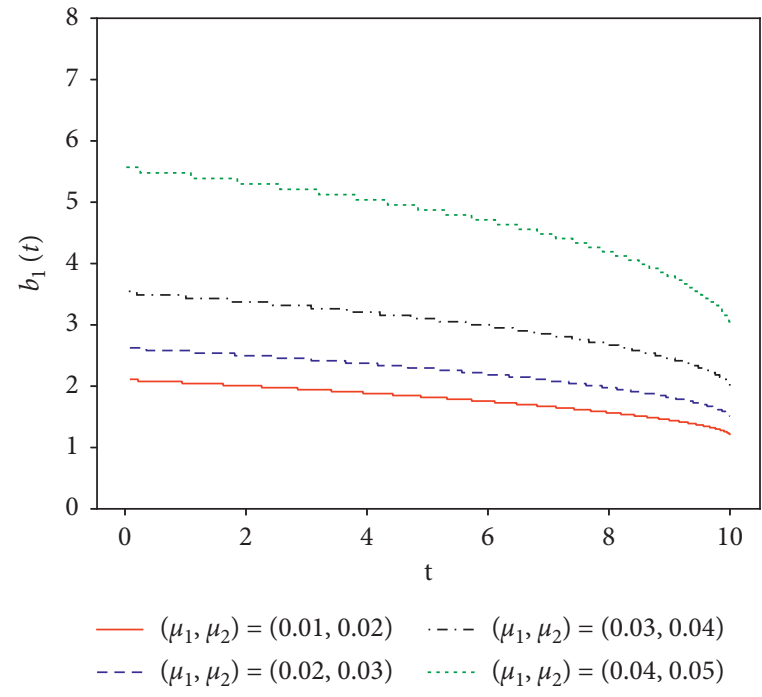

(a)

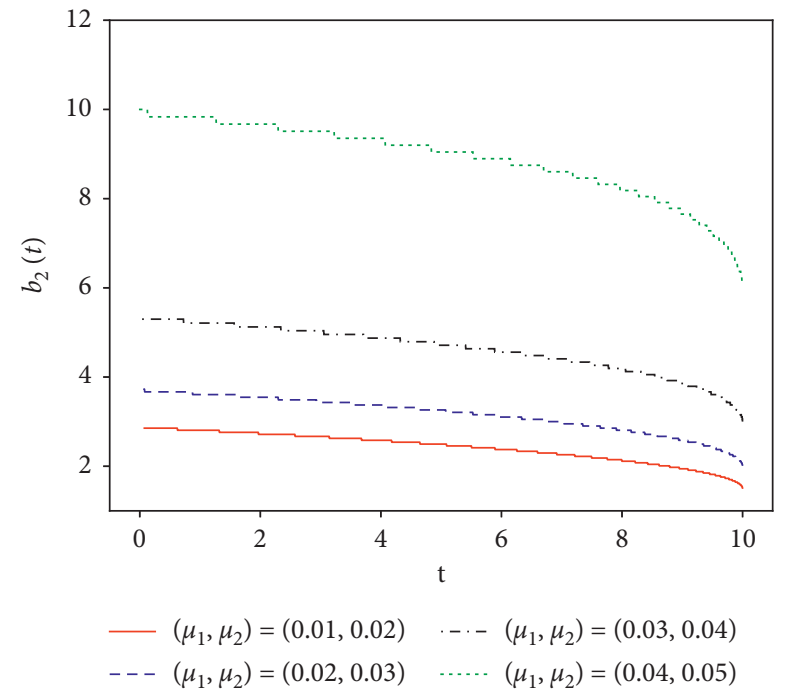

(b)

Figure 4: The impact of varying return rate of stock on the early exercise boundary for (A.) regime 1 and (B.) regime 2.

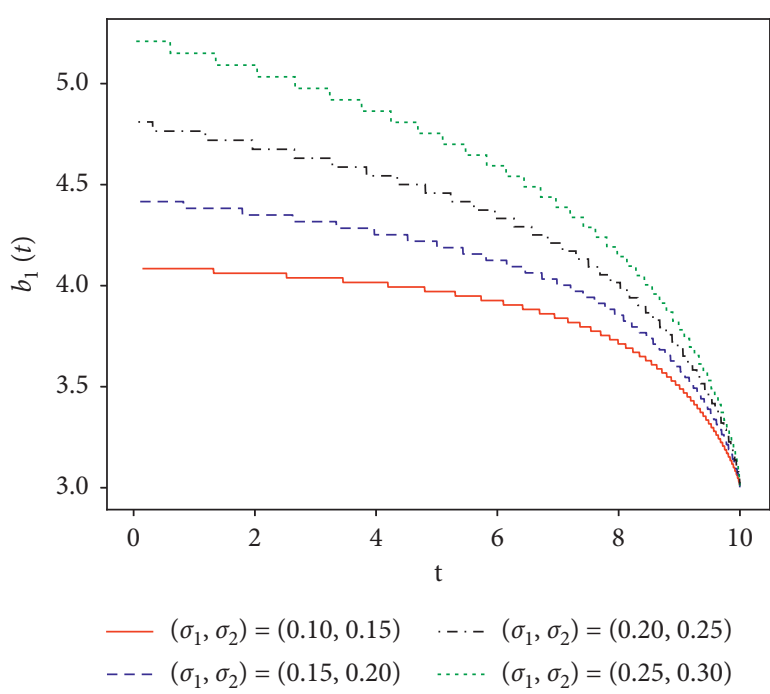

(a)

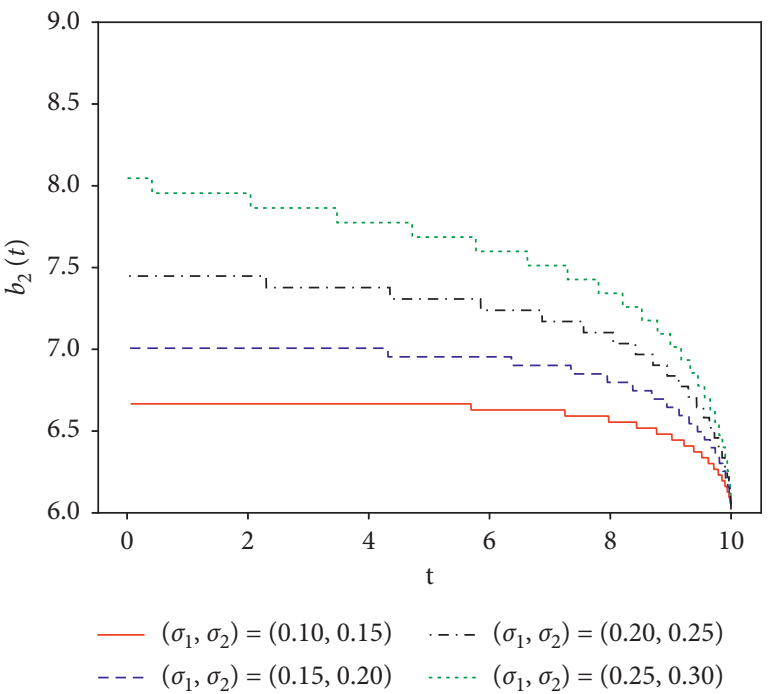

(b)

Figure 5: The impact of varying volatility on the early exercise boundary for (A.) regime 1 and (B.) regime 2.

assumed to be $T=1$. The transaction cost is assumed to be $a=0.4$. The interest rate is taken as $r=0.08$ for Figure $1(\mathrm{a})$ so that $r>\mu_{2}$ and $r=0.045$ for Figure 1(b) so that $\mu_{1}<\eta<r<\mu_{2}$. The number of time steps for both methods is $N=10000$. In Figure 1, we simulate the sample path of the stock price and plot the early exercise boundary of problem (4). Intuitively, from Figure 1(a), we see that if the risk-free interest rate is always higher than the return rate of stock, the stockholder will have motivation to sell the stock when the stock price hits the free boundary at time $t_{0}$. Hence, in this case, there exists a unique free boundary for each state. Moreover, the larger return rate and volatility mean that the value function is larger, and the early exercise boundary will be higher. Intuitively, from Figure 1(b), it is clear that if the risk-free interest rate is between the return rate of bear market and return rate of the bull market, then the stockholder will sell the stock early in bear market and hold the stock until the maturity date in bull market. Hence, in this case, there exists a unique free boundary when the investor is in bear market, and the free boundary does not exist when the investor is in bull market.

Example 2. In this example, we assess the impact of varying model parameters on the early exercise boundary.

Figure 2 displays the sensitivity with respect to transaction fee. We vary the transaction fee between 1 and 4 and compute the early exercise boundary. We observe that the early exercise boundary is higher for a higher transaction fee. 
The higher transaction fee means that the stockholder will sell the stock if stock price is higher. That is, the critical price will be higher.

In Figure 3, we show the sensitivity of the early exercise boundary with respect to interest rate. In our case, the interest rate takes values from 0.055 to 0.07 . The figure shows that with a higher interest rate, the early exercise boundary is lower. Intuitively, the higher interest rate motivates the stockholder to sell the stock although the stock price is lower.

Figure 4 illustrates the sensitivity with respect to the return rate of stock. For $\left(\mu_{1}, \mu_{2}\right)=$ $(0.01,0.02),(0.02,0.03),(0.03,0.04),(0.04,0.05)$, we compute the early exercise boundary. This figure shows that the free boundary is higher with a higher return rate. Intuitively, this result can be explained by the fact that the higher return rate makes the investor unwilling to sell the stock for a low stock price. Therefore, the critical price to sell stock is higher.

We analyze the sensitivity of the early exercise boundary with respect to the volatility in Figure 5. For $\left(\sigma_{1}, \sigma_{2}\right)=(0.10$, $0.15),(0.15,0.20),(0.20,0.25),(0.25,0.30)$, we compute the early exercise boundary. We observe that the higher the volatility, the higher is the early exercise boundary. Intuitively, if the volatility is higher, the stock price fluctuates more heavily and the investor will be more willing to hold the stock until the stock price goes up.

\section{Conclusions}

In this paper, we discuss the optimal selling rule under regime-switching models. To explore the optimal stopping rule, we analyze the optimal stopping problem and convert the problem into a free boundary problem. The properties of the free boundary and the involved Volterra type integral equations are established. Finally, we calculate the free boundary by trinomial tree method. Once the free boundary can be calculated, the optimal stopping rule is determined. We make a conclusion that, when the return of the stock is always higher than the interest rate, the agent should hold the stock until the maturity date; otherwise, he/she should sell the stock in the bear market when the stock price reaches a critical level and hold the stock until the maturity date in the bull market, which is consistent with intuition. Since the Volterra equations in this paper are interacting, this makes it difficult to solve the free boundary in each state independently. Therefore, the method of solving the Volterra equation without regime-switching cannot be extended to solve our problem directly. We will discuss this kind of problem in the future.

\section{Appendix}

\section{A. Proof of Lemma 1}

Proof. For any $s_{1}<s_{2}, \epsilon>0$, let $\tau_{s_{2}} \in\left[0, T-s_{2}\right]$ be the $\epsilon$-optimal stopping time for $V\left(s_{2}, x, i\right)$. It is clear that $0 \leq \tau_{s_{2}} \leq T-s_{1}$ is suboptimal for $V\left(s_{1}, x, i\right)$. This implies that

$$
V\left(s_{1}, x, i\right) \geq \mathbb{E}\left[e^{-r \tau_{s_{2}}} g\left(X_{\tau_{s_{2}}}^{x}\right)\right] \geq V\left(s_{2}, x, i\right)-\epsilon .
$$

Now, the monotonicity of $V$ in $t$ follows by letting $\epsilon \longrightarrow 0$.

For the monotonicity of $V$ in $x$, we solve the SDE (2) to obtain that

$$
X_{t}=x \exp \left(H_{t}\right)
$$

where $H_{t}$ is defined by (18). Now, by (4),

$$
V(t, x, i)=\sup _{\tau \in \Lambda_{0, T-t}} \mathbb{E}\left[e^{-r \tau}\left(x \exp \left(H_{\tau}\right)-a\right)\right], \quad i=1,2 .
$$

It is obvious that $V$ is increasing in $x$.

At last, we prove the convexity of $V(t, \cdot, i)$. For any $x_{1}, x_{2}>0, \lambda \in[0,1]$, we set $x^{*}=\lambda x_{1}+(1-\lambda) x_{2}$. (4) leads to

$$
\begin{aligned}
V\left(t, x^{*}, i\right) & =\sup _{\tau \in \Lambda_{0, T-t}} \mathbb{E}\left[e^{-r \tau}\left(X_{\tau}^{x^{*}}-a\right)\right] \\
& =\sup _{\tau \in \Lambda_{0, T-t}} \mathbb{E}\left[e^{-r \tau}\left(\lambda\left(X_{\tau}^{x_{1}}-a\right)+(1-\lambda)\left(X_{\tau}^{x_{2}}-a\right)\right)\right] \\
& \leq \lambda \sup _{\tau \in \Lambda_{0, T-t}} \mathbb{E}\left[e^{-r \tau}\left(X_{\tau}^{x_{1}}-a\right)\right]+(1-\lambda) \sup _{\tau \in \Lambda_{0, T-t}} \mathbb{E}\left[e^{-r \tau}\left(X_{\tau}^{x_{2}}-a\right)\right] \\
& =\lambda V\left(t, x_{1}, i\right)+(1-\lambda) V\left(t, x_{2}, i\right) .
\end{aligned}
$$

Hence, $V(t, \cdot, i)$ is a convex function.

\section{B. Proof of Lemma 2}

Proof. For the proof of Lipschitz continuity of $V(t, \cdot, i)$, we refer to [7]. It suffices to prove that $V(\cdot, x, i)$ is $1 / 2-$ Hölder continuous. The following argument is based on a similar method developed by [12]. Integrating the SDE (2) from 0 to $t$, we have

$$
X_{t}=x+\int_{0}^{t} \mu\left(\alpha_{s}\right) X_{s} \mathrm{~d} s+\int_{0}^{t} \sigma\left(\alpha_{s}\right) X_{s} \mathrm{~d} B_{s} .
$$

For any $0 \leq t_{1}<t_{2} \leq T$, we assume that $\tau_{1}$ is the $\epsilon$-optimal stopping time for $V\left(t_{1}, x, i\right)$. Then, $0 \leq \tau_{1} \leq T-t_{1}$, by setting $\tau_{2}=\tau_{1} \wedge\left(T-t_{2}\right)$. Then, it is clear that

$$
\begin{aligned}
0 \leq \tau_{1}-\tau_{2} & =-\left(\tau_{1} \wedge\left(T-t_{2}\right)-\tau_{1}\right)=-\left(0 \wedge\left(T-t_{2}-\tau_{1}\right)\right) \\
& \leq-\left(0 \wedge\left(T-t_{2}-T+t_{1}\right)\right)=t_{2}-t_{1} .
\end{aligned}
$$


Hence, we derive that

$$
\begin{aligned}
0 & \leq V\left(t_{1}, x, i\right)-V\left(t_{2}, x, i\right) \\
& \leq \mathbb{E}\left[e^{-r \tau_{1}}\left(X_{\tau_{1}}^{x}-a\right)\right]-\mathbb{E}\left[e^{-r \tau_{2}}\left(X_{\tau_{2}}^{x}-a\right)\right]+\varepsilon \\
& =\mathbb{E}\left[e^{-r \tau_{1}} X_{\tau_{1}}^{x}-e^{-r \tau_{2}} X_{\tau_{2}}^{x}\right]-a \mathbb{E}\left[e^{-r \tau_{1}}-e^{-r \tau_{2}}\right]+\varepsilon \\
& \leq \mathbb{E}\left[\left|X_{\tau_{1}}^{x}-X_{\tau_{2}}^{x}\right|\right]-a \mathbb{E}\left[e^{-r \tau_{1}}-e^{-r \tau_{2}}\right]+\varepsilon,
\end{aligned}
$$

where the second inequality follows from the fact that $\tau_{1}$ is $\epsilon$-optimal for $V\left(t_{1}, x, i\right)$ and $\tau_{2}$ is suboptimal for $V\left(t_{2}, x, i\right)$, the last inequality follows from (B.2). By mean value theorem and (B.2), it is obvious that

$$
\left|\mathbb{E}\left[e^{-r \tau_{1}}-e^{-r \tau_{2}}\right]\right| \leq C\left|t_{2}-t_{1}\right| .
$$

Applying (B.1) and (B.2) and Burkholder-Davis-Gundy inequality, we derive that

$$
\begin{aligned}
\mathbb{E}\left[\left|X_{\tau_{1}}^{x}-X_{\tau_{2}}^{x}\right|\right] & \leq \mathbb{E}\left[\left|\int_{\tau_{2}}^{\tau_{1}} \mu\left(\alpha_{s}\right) X_{s} \mathrm{~d} s\right|\right]+\mathbb{E}\left[\left|\int_{\tau_{2}}^{\tau_{1}} \sigma\left(\alpha_{s}\right) X_{s} \mathrm{~d} B_{s}\right|\right] \\
& \leq \mathbb{E}\left[\left|\int_{0}^{\tau_{1}-\tau_{2}} \mu\left(\alpha_{s+\tau_{2}}\right) X_{s+\tau_{2}} \mathrm{~d} s\right|\right]+\mathbb{E}\left[\left|\int_{0}^{\tau_{1}-\tau_{2}} \sigma\left(\alpha_{s+\tau_{2}}\right) X_{s+\tau_{2}} \mathrm{~d} B_{s}\right|\right] \\
& \leq \mu_{2} \mathbb{E}\left[\sup _{0 \leq t \leq T}\left|X_{t}\right|\right]\left(t_{2}-t_{1}\right)+\mathbb{E}\left[\sup _{0 \leq t \leq t_{2}-t_{1}}\left|\int_{0}^{t} \sigma\left(\alpha_{s+\tau_{2}}\right) X_{s+\tau_{2}} \mathrm{~d} B_{s}\right|\right] \\
& \leq \mu_{2} \mathbb{E}\left[\sup _{0 \leq t \leq T}\left|X_{t}\right|\right]\left(t_{2}-t_{1}\right)+C^{\prime} \mathbb{E}\left[\sqrt{\int_{0}^{t_{2}-t_{1}} \sigma^{2}\left(\alpha_{s+\tau_{2}}\right) X_{s+\tau_{2}}^{2} \mathrm{~d} s}\right] \\
& \leq \mu_{2} \mathbb{E}\left[\sup _{0 \leq t \leq T}\left|X_{t}\right|\right]\left(t_{2}-t_{1}\right)+C^{\prime} \sigma_{2} \mathbb{E}\left[\sup _{0 \leq t \leq T}\left|X_{t}\right|\right] \sqrt{t_{2}-t_{1}} .
\end{aligned}
$$

Now, since $\mathbb{E}\left[\sup _{0 \leq t \leq T}\left|X_{t}\right|\right] \leq C^{\prime \prime}(x, i, T)$ by moment estimates (see [18] or [12]), combining with (B.3)-(B.5), we conclude that $V$ is $1 / 2-$ Hölder continuous in $t$.

\section{Data Availability}

No data were used in this paper.

\section{Conflicts of Interest}

The authors declare that they have no conflicts of interest.

\section{Acknowledgments}

This work was supported by the Innovative Team Program of the Neijiang Normal University (Grant No. 2019TD02) and the Scientific Research Start-Up Program for Talents of Guizhou University of Finance and Economics (Grant No. 2019YJ070).

\section{References}

[1] H. P. McKean, "A free boundary problem for the heat equation arising from a problem in mathematical economics," Journal of Industrial Management Review, vol. 60, no. 9, pp. 32-39, 1965.

[2] J. Wang, M. Zhou, X. Jin, X. Guo, L. Qi, and X. Wang, "Variance minimization hedging analysis based on a timevarying Markovian DCC-GARCH model," IEEE Transactions on Automation Science and Engineering, vol. 17, no. 2, pp. 621-632, 2020.

[3] X. Hou, K. Wang, C. Zhong, Z. Wei, and " ST-Trader, "STtrader: a spatial-temporal deep neural network for modeling stock market movement," IEEE/CAA Journal of Automatica Sinica, vol. 8, no. 5, pp. 1015-1024, 2021.

[4] Q. Zhang, "Stock trading: an optimal selling rule," SIAM Journal on Control and Optimization, vol. 40, no. 1, pp. 67-84, 2001.

[5] P. Eloe, R. H. Liu, M. Yatsuki, G. Yin, and Q. Zhang, "Optimal selling rules in a regime-switching exponential Gaussian diffusion model," SIAM Journal on Applied Mathematics, vol. 69, no. 3, pp. 810-829, 2008.

[6] X. Guo and Q. Zhang, "Optimal selling rules in a regime switching model," IEEE Transactions on Automatic Control, vol. 50, no. 9, pp. 1450-1455, 2005.

[7] M. Pemy and Q. Zhang, "Optimal stock liquidation in a regime switching model with finite time horizon," Journal of Mathematical Analysis and Applications, vol. 321, no. 2, pp. 537-552, 2006.

[8] M. Pemy, "Optimal selling rule in a regime switching Lévy market," International Journal of Mathematics and Mathematical Sciences, vol. 2011, pp. 1-28, 2011.

[9] B. Bian, N. Wu, and H. Zheng, "Optimal liquidation in a finite time regime switching model with permanent and temporary pricing impact," Discrete and Continuous Dynamical Systems Series B, vol. 21, no. 5, pp. 1401-1420, 2016.

[10] J. Vaicenavicius, "Asset liquidation under drift uncertainty and regime-switching volatility," Applied Mathematics and Optimization, vol. 81, no. 3, pp. 757-784, 2020.

[11] J. Buffington and R. J. Elliott, "American options with regimeswitching," International Journal of Theoretical and Applied Finance, vol. 5, no. 5, pp. 497-514, 2002.

[12] S. D. Jacka and A. Ocejo, "On the regularity of American options with regime-switching uncertainty," Stochastic Processes and Their Applications, vol. 128, no. 3, pp. 803-818, 2018.

[13] S. Boyarchenkp and S. Levendorski, "American options in regime-switching models," SIAM Journal on Control and Optimization, vol. 48, no. 3, pp. 1353-1376, 2009. 
[14] Y. Liu and N. Privault, "Selling at the ultimate maximum in a regime switching model," International Journal of Theoretical and Applied Finance, vol. 20, no. 3, Article ID 1750018, 2017.

[15] X. Luo and J. Xing, "Optimal surrender policy of guaranteed minimum maturity benefits in variable annuities with regimeswitching volatility," Mathematical Problems in Engineering, vol. 2021, pp. 1-20, 2021.

[16] Z. Yang, "A system of variational inequalities arising from finite expiry Russian option with two regimes," Mathematical Methods in the Applied Sciences, vol. 32, no. 13, pp. 1681-1703, 2009.

[17] F. L. Yuen and H. Yang, "Option pricing with regime switching by trinomial tree method," Journal of Computational and Applied Mathematics, vol. 233, no. 8, pp. 1821$1833,2010$.

[18] X. Mao and C. Yuan, Stochastic Differential Equations with Markovian Switching, Imperial College Press, London, UK, 2006.

[19] G. Peskir and A. Shiryaev, Optimal Stopping and FreeBoundary Problems, Birkhauser, Basel, Switzerland, 2006.

[20] G. M. Lieberman, Second Order Parabolic Differential Equations, World Scientific, St. Hackensack, NJ, USA, 1996. 